\title{
DWARF SPHEROIDAL SATELLITES OF M31: I. VARIABLE STARS AND STELLAR POPULATIONS IN ANDROMEDA XIX*
}

\author{
FELICE CUSANO $^{1}$, GISELLA CLEMENTINI ${ }^{1}$, ALESSIA GAROFALO ${ }^{1,2}$, MICHELE \\ CIGNONI $^{2}$, LUCIANA FEDERICI ${ }^{1}$, MARCELLA MARCONI $^{3}$, ILARIA MUSELLA ${ }^{3}$, \\ VINCENZO RIPEPI ${ }^{3}$, KONSTANTINA BOUTSIA ${ }^{4}$, MARCO FUMANA ${ }^{5}$, STEFANO \\ GALLOZZI $^{4}$, VINCENZO TESTA $^{4}$ \\ ${ }^{1}$ INAF- Osservatorio Astronomico di Bologna, Via Ranzani 1, I - 40127 Bologna, Italy \\ felice.cusano@oabo.inaf.it, gisella.clementini@oabo.inaf.it, \\ luciana.federici@oabo.inaf.it \\ ${ }^{2}$ Dipartimento di Astronomia, Università di Bologna, Via Ranzani 1, I - 40127 Bologna, Italy \\ alessia.garofalo@studio.unibo.it, michele.cignoni@unibo.it \\ ${ }^{3}$ INAF- Osservatorio Astronomico di Capodimonte, Salita Moiariello 16, I- 80131 Napoli, Italy \\ marcella@na.astro.it, ilaria@na.astro.it, ripepi@na.astro.it \\ ${ }^{4}$ INAF- Osservatorio Astronomico di Roma, Via di Frascati 3300040 Monte Porzio Catone, Italy \\ konstantina.boutsia@oa-roma.inaf.it, stefano.gallozzi@oa-roma.inaf.it, \\ vincenzo.testa@oa-roma.inaf.it \\ ${ }^{5}$ INAF - IASF Milano, Via E. Bassini 15, I-20133 Milano, Italy \\ fumana@lambrate.inaf.it
}

\begin{abstract}
We present $B, V$ time-series photometry of Andromeda XIX (And XIX), the most extended (half-light radius of $6.2^{\prime}$ ) of Andromeda's dwarf spheroidal companions, that we observed with the Large Binocular Cameras at the Large Binocular Telescope. We surveyed a $23^{\prime} \times 23^{\prime}$ area centered on And XIX and present the deepest color magnitude diagram (CMD) ever obtained for this galaxy, reaching, at $V \sim 26.3 \mathrm{mag}$, about one magnitude below the horizontal branch (HB). The CMD shows a prominent and slightly widened red giant branch, along with a predominantly red $\mathrm{HB}$, which, however, extends to the blue to significantly populate the classical instability strip. We have identified 39 pulsating variable stars, of which 31 are of RR Lyrae type and 8 are Anomalous Cepheids (ACs). Twelve of the RR Lyrae variables and 3 of the ACs are located within And XIX's half light radius. The average period of the fundamental mode RR Lyrae stars $(\langle P a b\rangle=0.62 \mathrm{~d}, \sigma=0.03 \mathrm{~d})$ and the period-amplitude diagram qualify And XIX as an Oosterhoff-Intermediate system. From the average luminosity of the RR Lyrae stars $(\langle V(R R)\rangle$ $=25.34 \mathrm{mag}, \sigma=0.10 \mathrm{mag})$ we determine a distance modulus of $(\mathrm{m}-\mathrm{M})_{0}=24.52 \pm 0.23 \mathrm{mag}$ in a scale where the distance to the Large Magellanic Cloud (LMC) is $18.5 \pm 0.1$ mag. The ACs follow a well defined Period-Wesenheit $(P W)$ relation that appears to be in very good agreement with the $P W$ relationship defined by the ACs in the LMC.
\end{abstract}

Subject headings: galaxies: dwarf, Local Group — galaxies: individual (Andromeda XIX) —stars: distances — stars: variables: other - techniques: photometric 


\section{INTRODUCTION}

The number of satellites known to surround the Andromeda spiral galaxy (M31) has increased dramatically in the last few years thanks to the systematic imaging of the M31 halo being carried out by the Pan-Andromeda Archaeological Survey (PAndAS; Martin et al. 2006, 2009; Ibata et al. 2007; Irwin et al. 2008; McConnachie et al. 2008, 2009; Richardson et al. 2011), the Sloan Digital Sky Survey (SDSS; Zucker et al. 2004, 2007; Slater et al. 2011; Bell et al. 2011), and, lately, the Panoramic Survey Telescope and Rapid Response System 1 survey (Pan-STARRS1; Martin et al. 2013). The latest census of the M31 companions currently counts 31 dwarf spheroidal galaxies (dSphs) whose luminosities range from $10^{4}$ to $10^{8} L_{\odot}$. This number is expected to further grow as new diffuse stellar systems are being discovered in the M31 halo (e.g. PAndAS-48; Mackey et al. 2013) whose actual nature, whether extended globular clusters (GCs) or ultra-faint dwarfs (UFDs) like those discovered around the Milky Way (MW), remains to be established. In the framework of the hierarchic formation of structures the dSph satellites we observe today around M31 may be the survivors of Andromeda's building process. Their stellar content can thus provide insight to reconstruct the star formation history (SFH) and the merging episodes that led to the early assembling of the M31 halo. The synthetic modeling of deep color magnitude diagrams (CMDs) represents the most direct way for understanding the formation history of a galaxy. However, the CMDs currently available for the M31 dSphs generally sample only the brightest stars (e.g. Zucker et al. 2004; McConnachie et al. 2008; Richardson et al. 2011; Bell et al. 2011), and even when Hubble Space Telescope (HST) data are available (Pritzl et al. 2002, 2004, 2005; Mancone \& Sarajedini 2008; Yang \& Sarajedini 2012) they only reach slightly below the HB, as observing the main sequence turn-off (MSTO) of the oldest stellar populations at the distance of M31 $\left[(m-M)_{0}=24.47 \pm 0.07 \mathrm{mag}, \mathrm{D}=783 \mathrm{kpc}\right.$, McConnachie 2012, or $(m-M)_{0}=24.42 \pm 0.06$ mag, $\mathrm{D}=766 \mathrm{kpc}$, Federici et al. 2012 requires of the order of tens of HST orbits.

\footnotetext{
* Based on data collected with Large Binocular Cameras at the Large Binocular Telescope
}

The pulsating variable stars are a powerful alternative tool to investigate the different stellar generations occurred in the M31 dSphs, as Classical Cepheids can be used to trace the young stars (typical ages ranging from a few to a few hundred Myr), and the RR Lyrae stars, which are comparably old but about 3 magnitude brighter than the MSTO, can allow to unravel the oldest stars born more than 10 Gyr ago. Using characteristics like the mean period of the fundamental-mode (RRab) and first-overtone (RRc) pulsators and the ratio of number of RRc $\left(N_{c}\right)$ to total number of RR Lyrae stars $\left(N_{a b+c}\right)\left(f_{c}=N_{c} / N_{a b+c}\right)$ the MW field and cluster RR Lyrae are divided into two different groups (Oosterhoff 1939): Oosterhoff type I (Oo I) clusters have $\left\langle P_{\mathrm{ab}}\right\rangle \simeq 0.55 \mathrm{~d}$, $\left\langle P_{\mathrm{c}}\right\rangle \simeq 0.32 \mathrm{~d}$, and $f_{c} \sim 0.17$; and Oosterhoff type II (Oo II) clusters have $\left\langle P_{\mathrm{ab}}\right\rangle \simeq 0.65 \mathrm{~d},\left\langle P_{\mathrm{c}}\right\rangle \simeq$ $0.37 \mathrm{~d}$, and $f_{c} \sim 0.44$. Different Oosterhoff types also imply slightly different ages and metallicities (van den Bergh 1993), as Oo II clusters are more metal-poor and older than Oo I systems. Recent studies (see e.g. Catelan 2009, for a review) confirmed the Oosterhoff dichotomy to occur not only among the MW GCs, but also for field variable stars in the MW halo. On the other hand, the separation in Oosterhoff groups seems to be a characteristic of the MW, since the dSphs around our galaxy, as well as their respective GCs, have $0.58 \leq\left\langle\mathrm{P}_{\mathrm{ab}}\right\rangle \leq 0.62 \mathrm{~d}$, and Oo-Intermediate (OoInt) properties (Catelan 2009; Clementini et al. 2010, and references therein). The characterization of the RR Lyrae population in M31 and in its companions is still at an early stage. In a deep survey of the M31 halo, Brown et al. (2004) identified 55 RR Lyrae stars (29 RRab, 25 RRc, and one double-mode, RRd, pulsator) in a $3.5^{\prime} \times 3.7^{\prime}$ field along the southeast minor axis of the galaxy. Based on their pulsation properties, Brown et al. (2004) concluded that unlike in the MW the old population in the M31 halo has Oo-Int properties. However, a different conclusion was reached by Sarajedini et al. (2009) who identified $681 \mathrm{RR}$ Lyrae variables (555 RRab's, and 126 RRc's) based on the HST/Advanced Camera for Surveys (ACS) observations of two fields near M32, at a projected distance between 4 and $6 \mathrm{kpc}$ from the center of M31, and concluded that these M31 fields have Oo-I properties. A total of $108 \mathrm{RR}$ Lyrae stars were identified by Jeffery et al. (2011) 
in six HST/ACS ultra-deep fields located in the disk, stream and halo of M31, showing that the RR Lyrae population appears mostly to be of the Oo-I and Oo-Int types. Of the M31 globular clusters only two had the RR Lyrae stars fully characterized, B514 was found to have Oo-Int properties (Clementini et al. 2009) and G11 to be an Oo II GC (Contreras Ramos et al. 2013). Six of the M31 dSphs have been analysed so far for variability and RR Lyrae stars have been identified in all of them (Pritzl et al. 2002, 2004, 2005; Mancone \& Sarajedini 2008; Yang \& Sarajedini 2012). According to these studies all three Oosterhoff types (Oo I, Oo II, and Oo-Int) seem to be present among the M31 satellites. These previous studies of variables in the M31 dSphs are based on Wide Field Planetary Camera 2 onboard the HST data. However, the HB of the M31 satellites can easily be reached from the ground with 8-10 $\mathrm{m}$ class telescopes. The ground-based facilities usually allow to cover areas significantly larger than the half-light radius $\left(r_{h}\right)$ of the M31 satellites, which often are rather extended, thus attaining much complete and statistically significant samples. We have obtained multi-band photometry of a sample of the M31 dSph satellites (see Clementini et al. 2011) using the Large Binocular Telescope (LBT) and the Gran Telescopio Canarias (GTC) and in this paper, we present results from our study of the stellar population and variable stars in Andromeda XIX (McConnachie et al. 2008), the most extended of Andromeda's dSph companions.

Andromeda XIX (And XIX, R.A. $=00^{h} 19^{m} 32.1^{s}$, DEC. $=+35^{\circ} 02^{\prime} 37.1^{\prime \prime}, \mathrm{J} 2000.0 ; \mathrm{l}=115.6^{\circ}, \mathrm{b}=-27.4^{\circ}$; McConnachie et al. 2008) was discovered by McConnach (2008) in a photometric survey of the southwestern quadrant of M31 performed with the Megaprime camera of the Canada-France-Hawaii Telescope (CFHT). The galaxy is located at a projected distance of $\sim 120 \mathrm{kpc}$ (Conn et al. 2012) from the center of M31. The discovery data show a steep red giant branch (RGB) but do not reach deep enough to sample the galaxy HB. McConnachie et al. (2008) estimate for And XIX an heliocentric distance of $933 \mathrm{kpc}$ using the luminosity of the RGB tip, however a closer distance of $821 \mathrm{kpc}$ is derived by Conn et al. (2012) applying to the same data a bayesian approach to estimate the luminosity of the RGB tip. With a half-light radius of $r_{h}=6.2^{\prime}$ (corresponding to a linear extension of $\sim 1.7$ or $1.5 \mathrm{kpc}$ whether McConnachie et al. 2008 or Conn et al. 2012 distance estimate is adopted) And XIX is the largest of Andromeda's dSph companions as well as the most extended of the Local Group (LG) satellites. In the stellar density map presented by McConnachie et al. (2009) an overdensity of stars named Southwest Cloud seems to connect And XIX with the halo of M31, providing hints of a possible interaction between And XIX and Andromeda itself. Collins et al. (2013) investigated spectroscopically 27 stars located around the RGB of And XIX measuring a small velocity dispersion when compared to the galaxy radial extent. The authors attributed this "cold" velocity dispersion to the tidal interaction with M31. From the spectra they also derived an average value of the metallicity $[\mathrm{Fe} / \mathrm{H}]=-1.8 \pm 0.3$ dex using the equivalent width of the calcium triplet and the Starkenburg et al. (2010) method, which is consistent with the value of $[\mathrm{Fe} / \mathrm{H}]=-1.9 \pm 0.1$ dex found photometrically by McConnachie et al. (2008) by isochrone-fitting of the galaxy CMD.

The paper is organized as follow: observations, data reduction and calibration of And XIX photometry are presented in Section 2. Results on the identification and characterization of the variable stars, the catalog of light curves, and the Oosterhoff classification of the RR Lyrae stars are discussed in Sections 3 and 4. The distance to And XIX derived from the RR Lyrae stars is presented in Section 5. The galaxy CMD is presented in Section 6. Properties and classification of the variable stars above the HB are discussed in Secetoal.'. In Section 8 an estimate of the contamination from the halo of M31 is given. The discussion on the spatial distribution of the And XIX's stars is presented in Section 9. In Section 10 we give an interpretation of the CMD using stellar isochrones and evolutionary tracks. Finally, a summary of the main results is presented in Section 11.

\section{OBSERVATIONS AND DATA RE- DUCTIONS}

Time series $B, V$ photometry of And XIX was obtained in fall 2010 using the Large Binocular Cameras (LBC1 $)$ mounted at the foci of the LBT.

${ }^{1}$ See http : //lbc.oa - roma.inaf.it/ 
Each LBC consists of an array of 4 CCDs with total field of view (FoV) of $\sim 23^{\prime} \times 23^{\prime}$ and pixel scale of 0.225 "/pixel. The two LBCs are optimized for the blue and red portion of the visible spectrum. The $B$ exposures were obtained with the Blue LBC, whereas the $V$ exposures were obtained with the Red LBC. We obtained $44 \mathrm{~B}$ and $31 \mathrm{~V}$ images each corresponding to a $420^{s}$ exposure, for total exposure times of $18480^{s}$ and $13020^{s}$ in $B$ and $V$, respectively. The log of And XIX observations is provided in Table 1

Each image was pre-reduced (bias-subtracted, flat-fielded and astrometrized) by the LBT team through the LBC dedicated pipeline. PSF photometry was then performed using the DAOPHOTALLSTAR-ALLFRAME package (Stetson 1987, 1994). The alignment of the images was performed using DAOMATCH, one of the routines in the DAOPHOT package, whereas DAOMASTER (Stetson 1992) was used to match point sources. The absolute photometric calibration to Johnson $B$ and $V$ was performed using stars in the scientific fields, for which calibrated photometry is available from previous studies. As a first step, we crossmatched our photometric catalogs composed by sources in the four CCDs with the SDSS catalog (Abazajian et al. 2009). The SDSS cata$\log$ was queried only for objects flagged as stars by the reduction package, and with good quality of the observations. A total of 1121 stars were found to be common between the two catalogs. The calibration equations available at http://www.sdss.org/dr4/algorithms/sdssUBVRI Transform.html were used to convert the $g$ and $r$ magnitudes of the SDSS stars to the Johnson $B$ and $V$ magnitudes. The parameters of the photometric calibration were finally derived fitting the data to the equations $B-b=\mathrm{c}_{B}+\mathrm{m}_{B} \times(b-v)$ and $V-v=\mathrm{c}_{V}+\mathrm{m}_{V} \times(b-v)$, where $B$ and $V$ are the standard Johnson magnitudes of the SDSS stars, and $b$ and $v$ are the instrumental magnitudes in our LBC catalog. The fit was performed using a $3 \sigma$ clipping rejection algorithm. A total of 512 stars were used in the final fit, with magnitudes ranging from 15.7 to $24.3 \mathrm{mag}$ in $B$, and from 0.2 to $1.7 \mathrm{mag}$ in the $B-V$ color 2 . The final r.m.s. of the fit is of 0.04 mag both in $B$ and $V$.

\footnotetext{
${ }^{2}$ The coefficients of the calibration equations are:
} $\mathrm{c}_{B}=27.532, \mathrm{~m}_{B}=-0.137, \mathrm{c}_{V}=27.530, \mathrm{~m}_{V}=-0.0633$

\section{IDENTIFICATION OF THE VARI- ABLE STARS}

The first step in the identification of variable stars was to search for objects with higher values of the variability index computed in DAOMASTER (Stetson 1994). We then also checked for variability all the stars with colors and magnitudes falling within the edges of the RR Lyrae and AC instability strips (ISs), according to the definition by Marconi et al. (2004). The final list of candidate variable stars consisted of $\sim 300$ sources that all were inspected visually using the Graphical Analyzer of Time Series package (GRaTIS), custom software developed at the Bologna Observatory by P. Montegriffo (see, e.g., Clementini et al. 2000). GRaTiS uses both the Lomb periodogram (Lomb 1976; Scargle 1982) and the best fit of the data with a truncated Fourier series (Barning 1963). The majority of the candidate variables were identified in both photometric bands, however, in few cases we have a reliable light curve only in the $B$ band. The period search was performed first on the $B$ band for which we have a larger number of epochs (44 phase-points). Final periods were then derived through an iterative procedure between the two photometric bands. The depth and sampling of the $B$ data is such that we were able to detect variables as faint as $B \sim 26 \mathrm{mag}$, with periods ranging from a few hours to several days. A total of 39 bona-fide variable stars were identified, of which 31 are RR Lyrae stars (23 RRab's, and 8 RRc's) lying on the galaxy HB, and 8 were classified as ACs on the basis of the luminosities about 1-1.5 mag brighter than the HB level, and the comparison with the AC IS, theoretical isochrones, and the Period-Wesenheit $(P W)$ relation (see Sects. 7, and 10.2). Among them are 5 RR Lyrae stars and $1 \mathrm{AC}$ for which we have reliable data only in the $B$ band. Identification and properties of the confirmed variable stars are summarized in Table 2. Column 1 gives the star identifier. We assigned to the variables an increasing number starting from the galaxy center, for which we adopted McConnachie et al. (2008)'s coordinates. Columns 2 and 3 provide the right ascension and declination (J2000 epoch), respectively. These coordinates were obtained from our astrometrized catalogs. Column 4 gives the type of variability. Columns 5 and 6 list the pulsation period and the Heliocentric Julian Day (HJD) of 

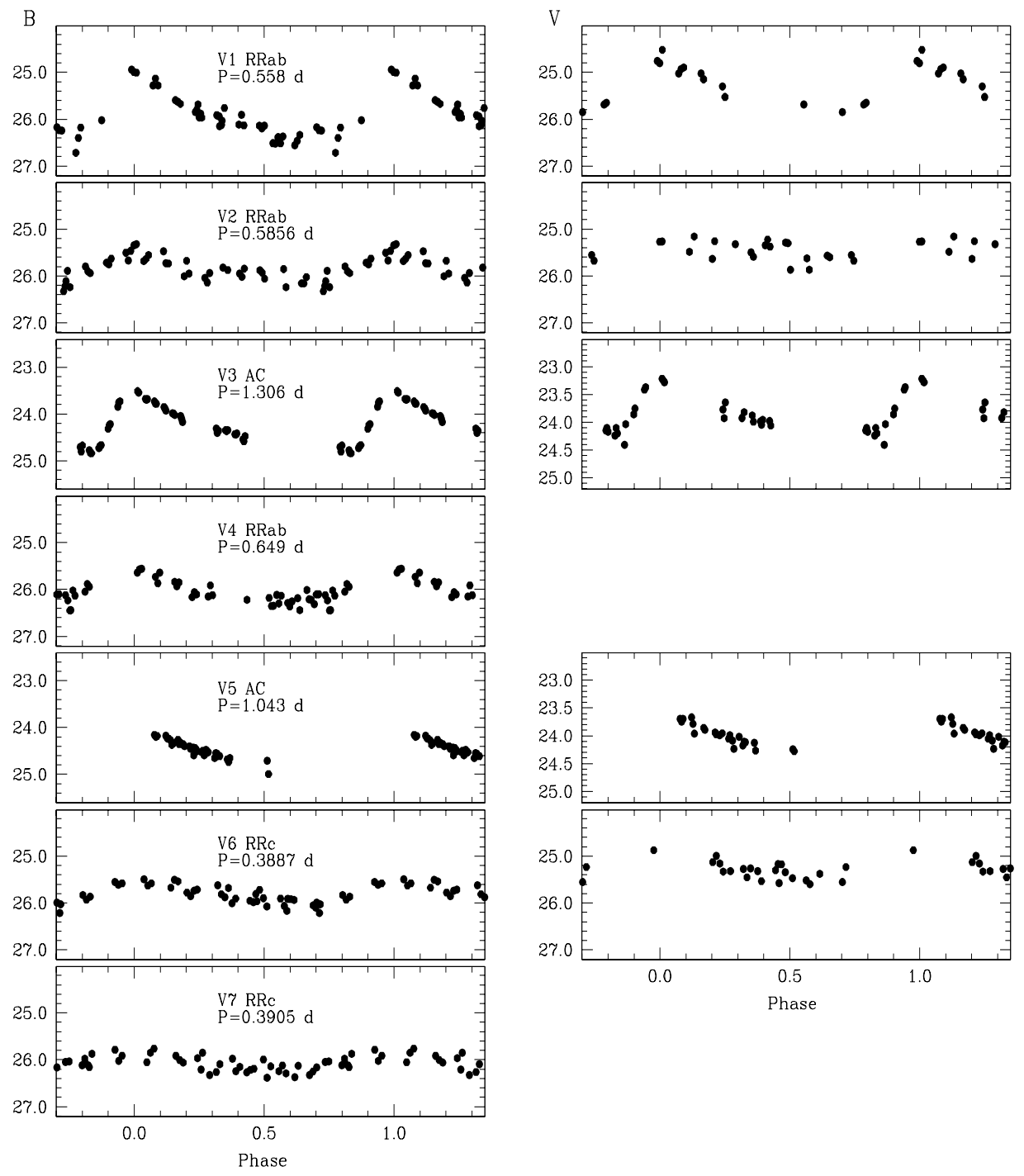

Fig. 1.-B (left panels) and $V$ (right panels) light curves of the variable stars identified in And XIX. Stars are ordered with increasing distance from the galaxy center, for which we adopted the McConnachie et al. (2008) coordinates. Typical internal errors for the single-epoch data are in the range of 0.04 to $0.12 \mathrm{mag}$ in $B$, and of 0.08 to 0.19 mag in $V$. 

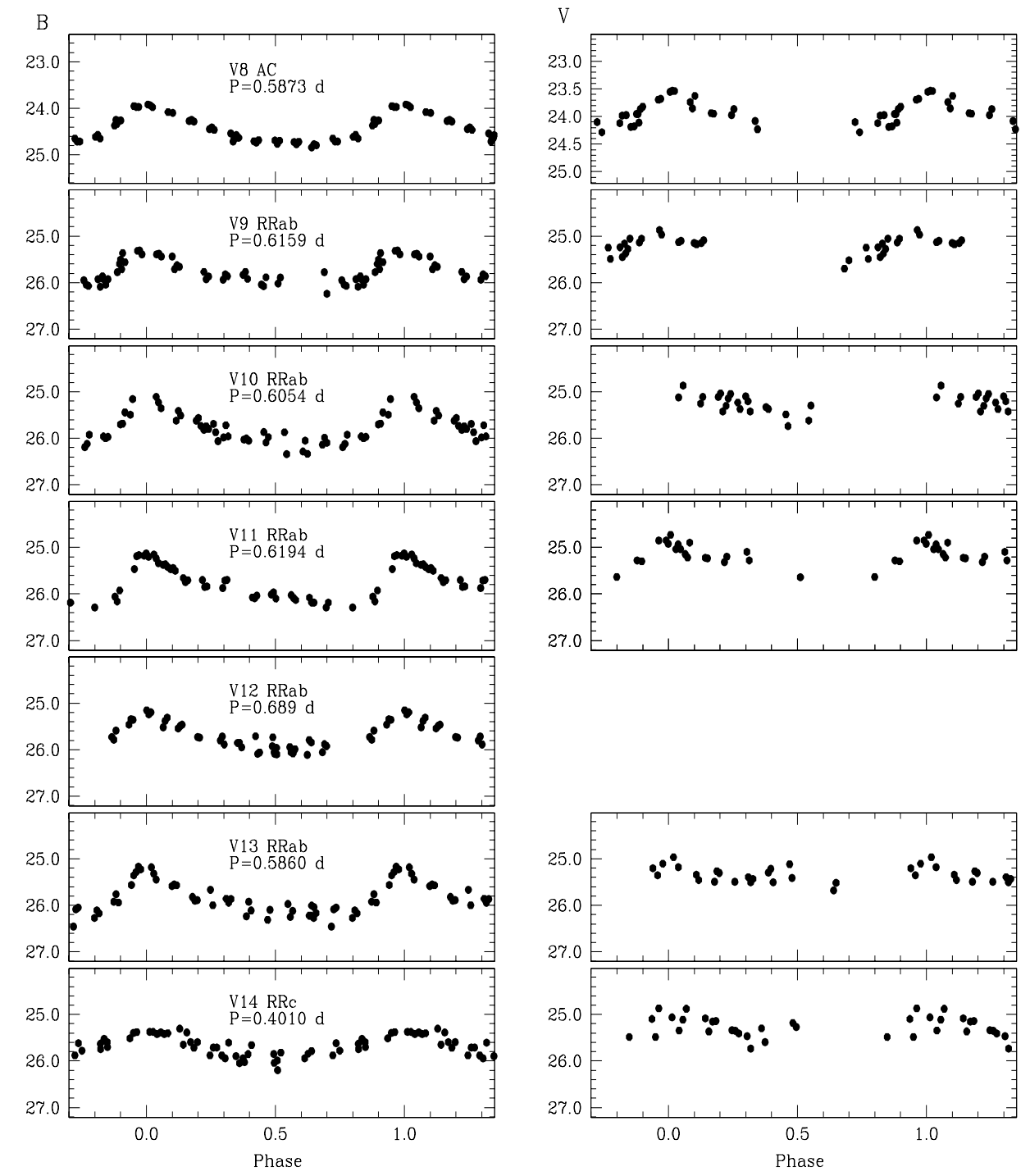

Fig. 1. - continued - 


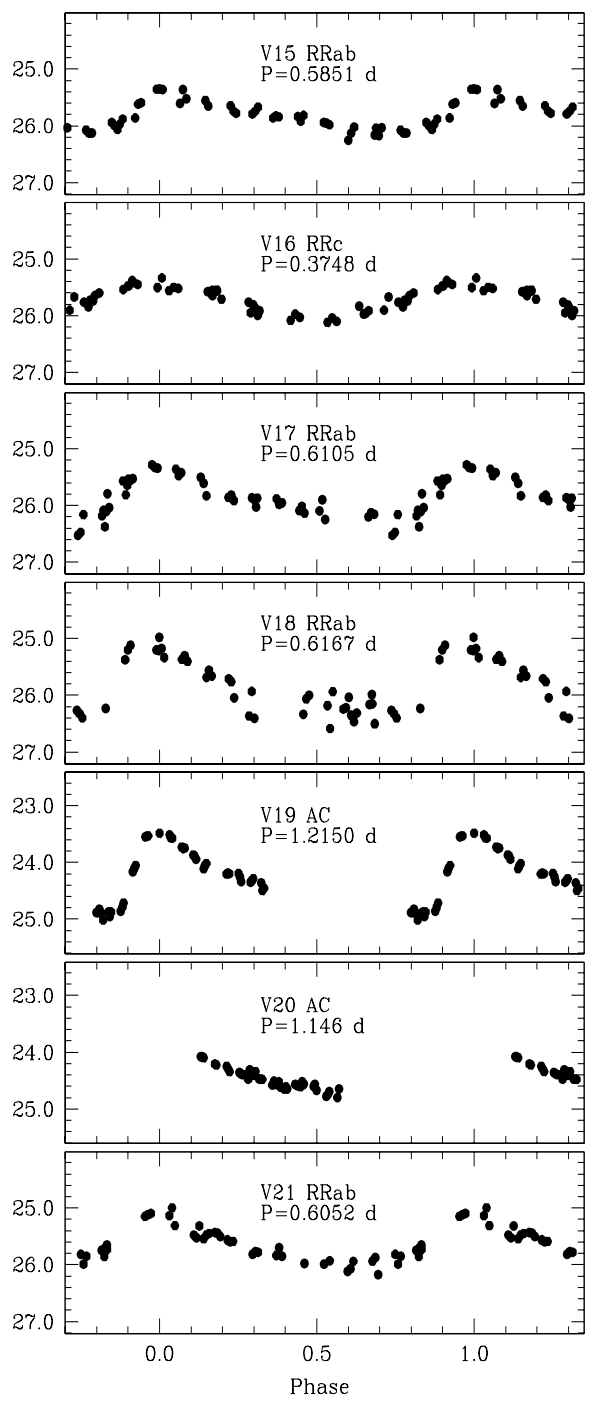

V

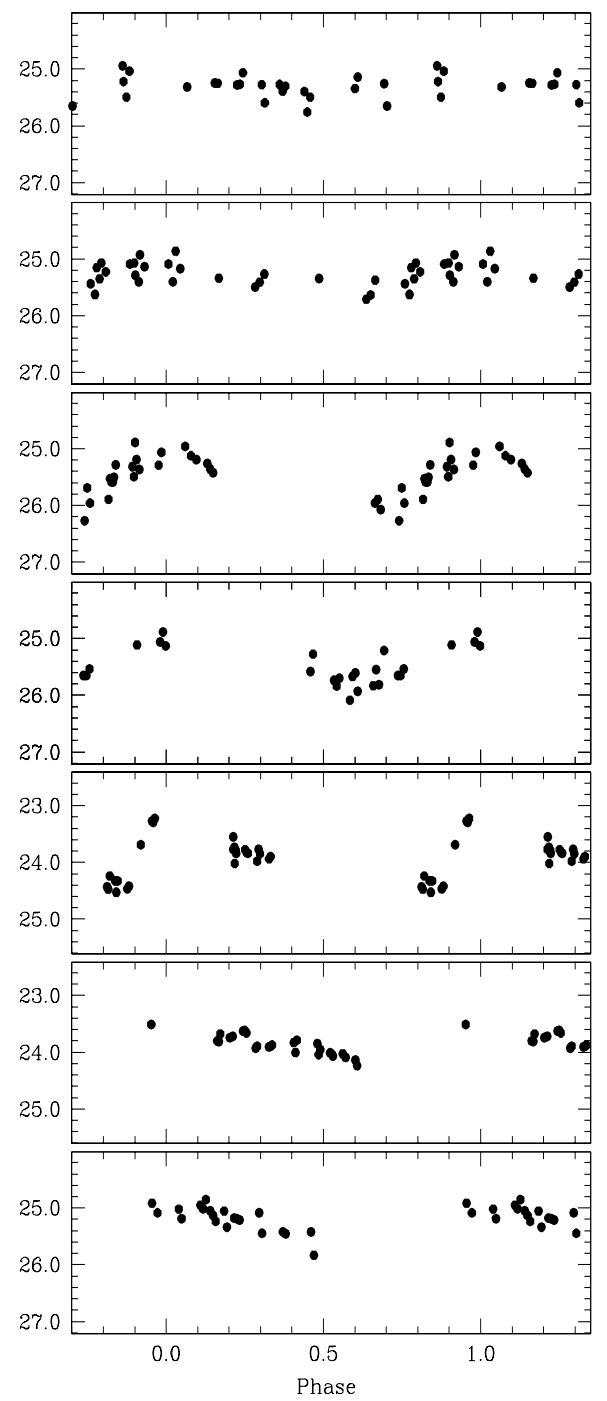

Fig. 1.- continued - 


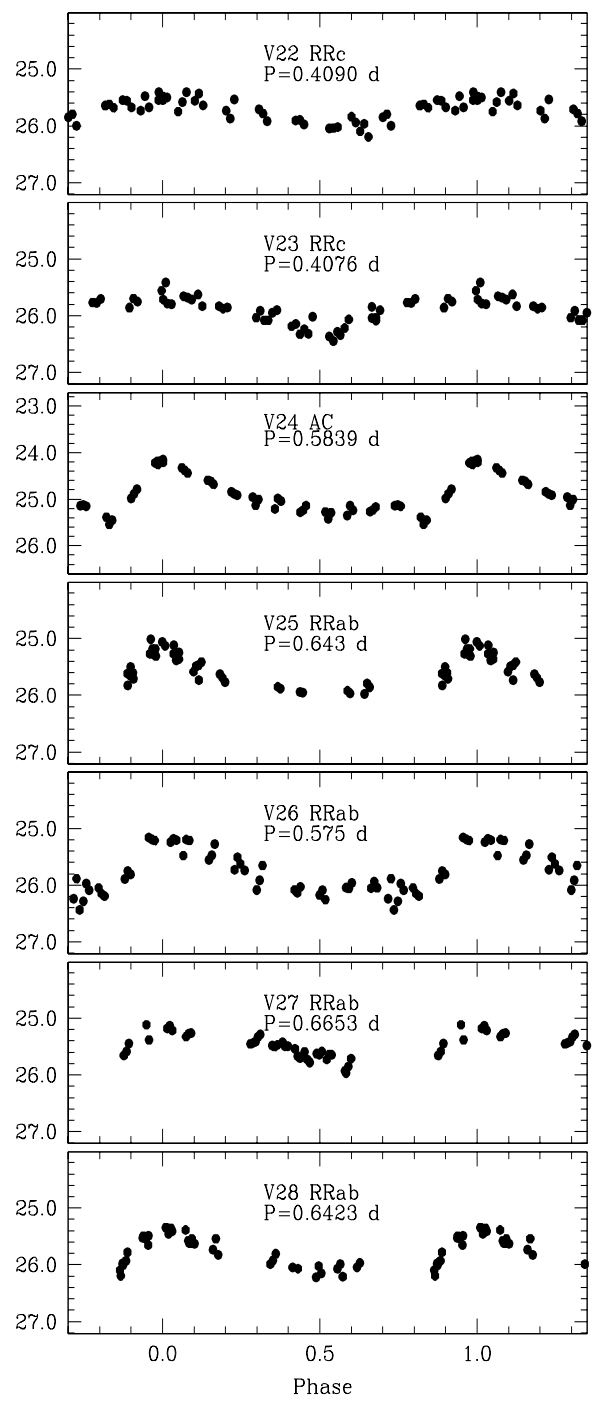

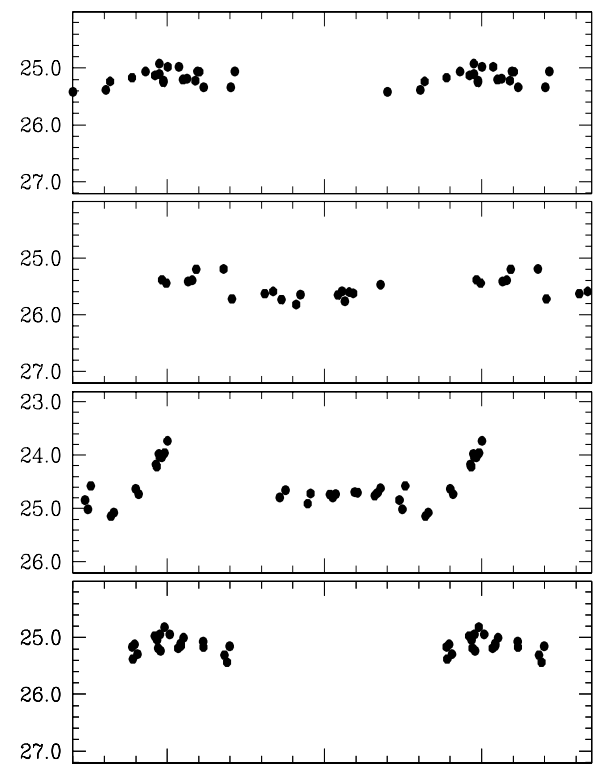

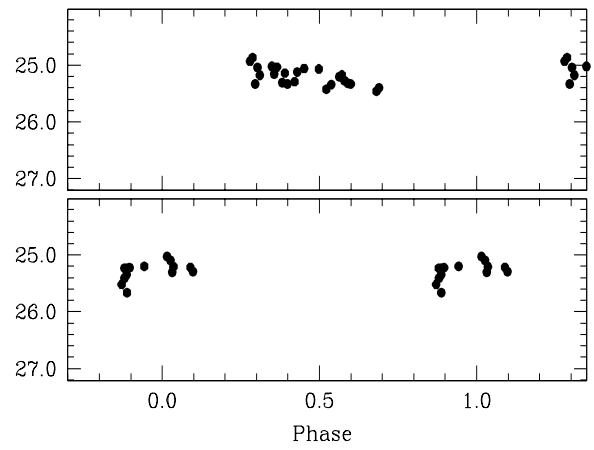

Fig. 1. - continued - 
B

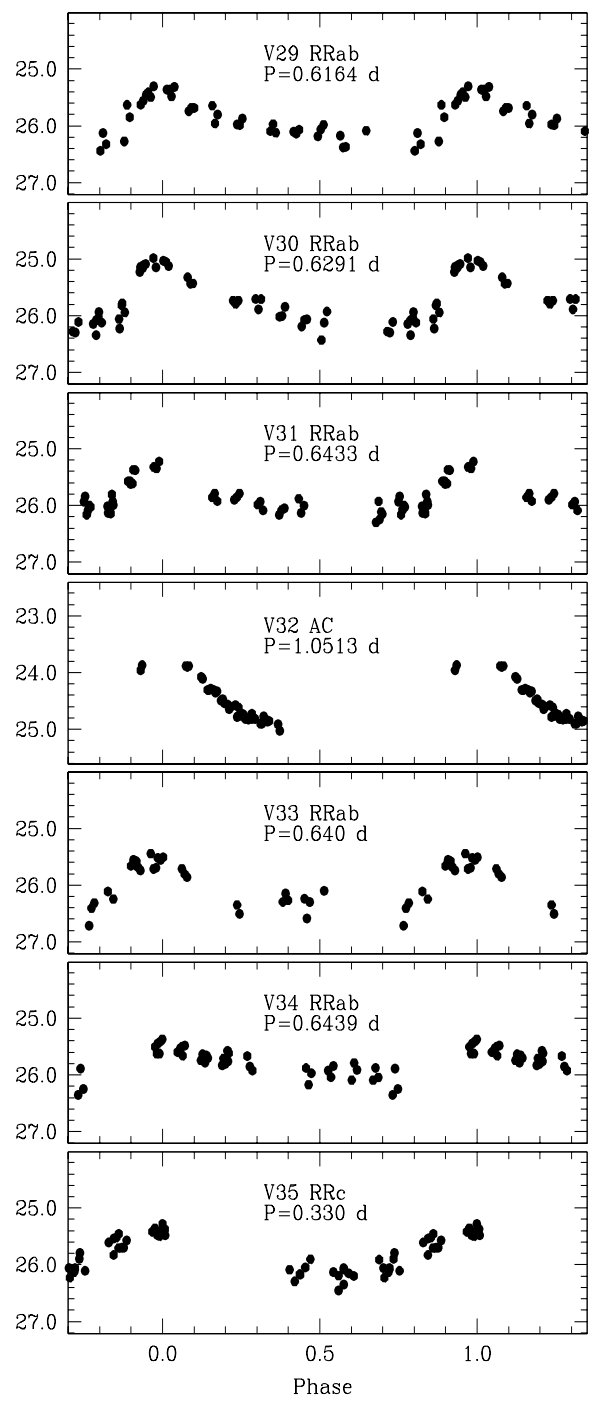

V

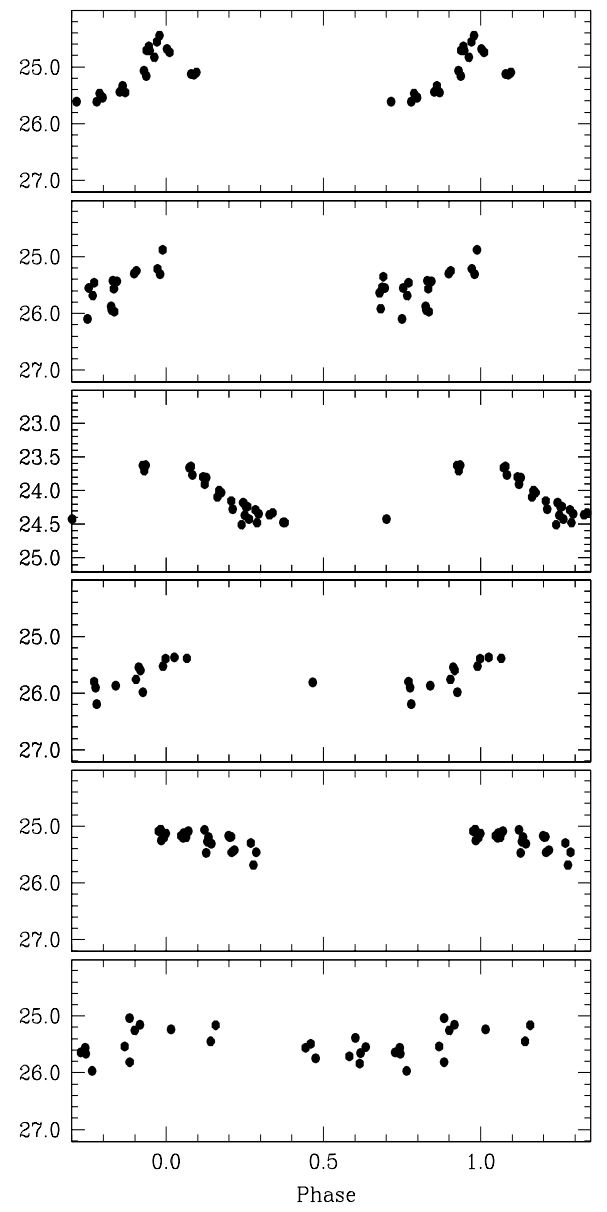

Fig. 1. - continued - 
Table 1: Log of And XIX observations

\begin{tabular}{lcccc}
\hline \hline Dates & Filter & $\mathrm{N}$ & $\begin{array}{c}\text { Exposure time } \\
(\mathrm{s})\end{array}$ & $\begin{array}{c}\text { Seeing (FWHM) } \\
(\operatorname{arcsec})\end{array}$ \\
\hline October 8, 2010 & $B$ & 2 & 420 & 1.5 \\
December 1-3, 2010 & $B$ & 42 & 420 & 0.7 \\
& & & & \\
October 8-11, 2010 & $V$ & 6 & 420 & $1.3-2.0$ \\
December 1, 2010 & $V$ & 25 & 420 & 0.8 \\
\hline
\end{tabular}

B

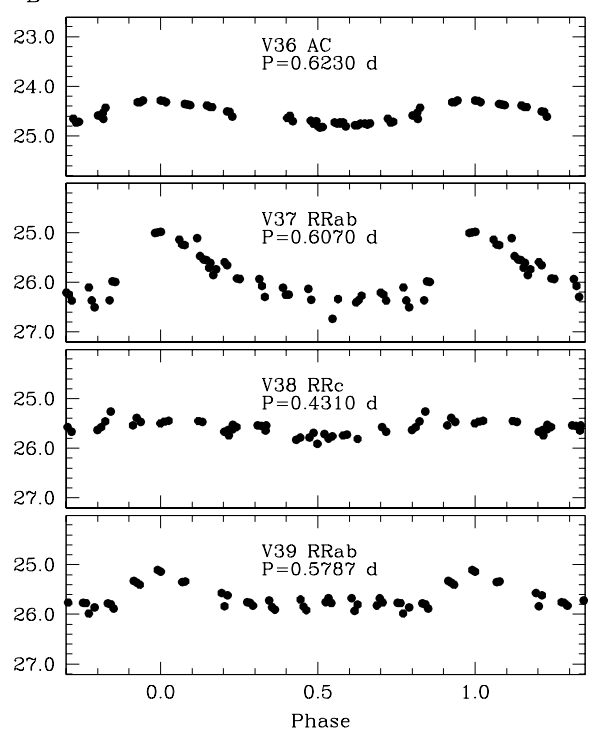

V

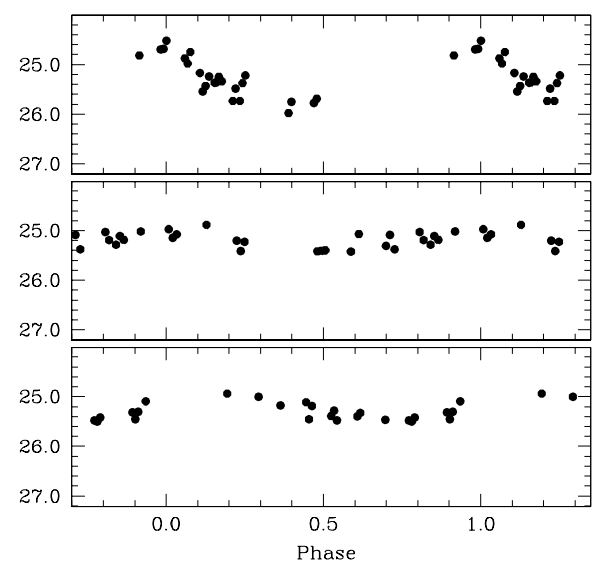

Fig. 1.- continued - 
maximum light, respectively. Columns 7 and 8 give the intensity-averaged mean $B$ and $V$ magnitudes, while Columns 9 and 10 list the corresponding amplitudes of the light variation. Light curves for the 39 stars are shown in Figure 1. To calibrate the photometry of stars that only have $B$ light-curves we simply added to the instrumental photometry the zero point $\mathrm{c}_{B}$ of the $B$ calibration equation. The time-series photometry of the variable stars is provided in Table 3, which is published in its entirety in the electronic edition of the Journal.

Figure 2 shows the position of the variable stars in the FoV covered by our LBC observations. In the figure black dots mark non-variable stars, red circles and blue triangles mark RRab and RRc stars, respectively, while the ACs are shown by green squares. A black ellipse drawn by convolving And XIX half-light radius with the galaxy ellipticity and position-angle, as published by McConnachie et al. (2008), delimitates the region containing the bulk of And XIX's stars. In the following we refer to this area as the region within And XIX half-light radius.

\section{PERIOD-AMPLITUDE DIAGRAM AND OOSTERHOFF CLASSIFICA- TION}

The right panel of Figure 3 shows the $B$-band period-amplitude diagram (also known as Bailey diagram, Bailey 1902) of the RR Lyrae stars identified in this study. Open symbols mark variable stars falling inside the ellipse shown in Figure2. Variables inside and outside the galaxy halflight radius do not show differences in this plot. Also shown in the figure are the loci defined, respectively, by the bona-fide regular (solid curves) and the well-evolved (dashed curves) fundamentalmode RR Lyrae stars in the Galactic globular cluster (GC) M3, from Cacciari et al. (2005). M3 has Oo I properties and its regular RRab stars define very well the locus occupied by the MW Oo I GCs, whereas the evolved RRab stars mimic the locus defined by the MW Oo II GCs. All the RR Lyrae stars identified in this study, independently of being located inside or outside And XIX's half-light radius, fall on either the Oo I locus, or between the two lines defined by the M3 variables (Cacciari et al. 2005). The left panel of
Figure 3 shows the comparison of And XIX variables with the period-amplitude diagram of the RR Lyrae stars detected in other four M31 dwarf satellites (namely, And I, And II, And III and And VI). Data for the RR Lyrae stars in And I and And III are from Pritzl et al. (2005), for And II from Pritzl et al. (2004), and for And VI from Pritzl et al. (2002). The variables appear to concentrate mainly towards the Oo-I locus or in the region between Oo I and Oo-Int lines, thus suggesting that there is paucity of Oo-II satellites around M31.

The average period of the RRab stars inside the ellipse in Figure 2 is $\langle\mathrm{Pab}\rangle=0.610 \mathrm{~d}$ (average on 7 stars, $\sigma=0.03 \mathrm{~d}$ ) and becomes $\langle\mathrm{Pab}\rangle=$ $0.62 \mathrm{~d}$ (average on 23 stars, $\sigma=0.03 \mathrm{~d}$ ) if we average over all the RRab stars identified in the present study. This along with the position on the period-amplitude diagram suggest that all the RR Lyrae stars we have identified likely belong to And XIX. The ratio of number of RRc over total number of RR Lyrae stars is $f_{c}=0.26$. Based on these evidences we conclude that And XIX has Oo-Int properties.

\section{DISTANCE}

We measured the distance to And XIX using its RR Lyrae stars. The average $V$ magnitude of the RR Lyrae stars is $\langle\mathrm{V}(\mathrm{RR})\rangle=25.34 \mathrm{mag}$ ( $\sigma=0.10 \mathrm{mag}$, average over 26 stars $)$. If we consider only RR Lyrae stars inside the galaxy half-light radius (ellipse in Fig. 22 namely, the 7 RRab stars: V1, V2, V4, V9, V10, V11, V12; and the two RRc stars V6, V7) the average becomes $\langle\mathrm{V}(\mathrm{RR})\rangle=25.32 \mathrm{mag}(\sigma=0.07 \mathrm{mag}$, average over 9 stars). The difference between the two average values is negligible, thus further confirming that all the RR Lyrae stars we have identified likely belong to And XIX. In the following we will use the average over all the RR Lyrae stars as more representative of the whole galaxy. The $\langle\mathrm{V}(\mathrm{RR})\rangle$ value was de-reddened using a standard extinction law $\left(\mathrm{A}_{\mathrm{V}}=3.1 \times \mathrm{E}(\mathrm{B}-\mathrm{V})\right)$ and as a first approach the reddening value $\mathrm{E}(\mathrm{B}$ $\mathrm{V})=0.066 \pm 0.026 \mathrm{mag}$ provided by Schlegel et al. (1998) maps. We then assumed an absolute magnitude of $\mathrm{M}_{\mathrm{V}}=0.54 \pm 0.09 \mathrm{mag}$ for RR Lyrae stars at $[\mathrm{Fe} / \mathrm{H}]=-1.5 \operatorname{dex}$ (Clementini et al. 2003; this is consistent with a distance modulus for the Large 
Table 2: Identification and properties of the variable stars identified in And XIX

\begin{tabular}{|c|c|c|c|c|c|c|c|c|c|}
\hline Name & $\begin{array}{c}\alpha \\
(2000)\end{array}$ & $\begin{array}{c}\delta \\
(2000)\end{array}$ & Type & $\begin{array}{c}\mathrm{P} \\
\text { (days) }\end{array}$ & $\begin{array}{l}\text { Epoch }(\max ) \\
\text { JD }(2400000)\end{array}$ & $\begin{array}{l}\langle\mathrm{B}\rangle \\
\text { (mag) }\end{array}$ & $\begin{array}{c}\langle\mathrm{V}\rangle \\
(\mathrm{mag})\end{array}$ & $\begin{array}{c}\mathrm{A}_{B} \\
(\mathrm{mag})\end{array}$ & $\begin{array}{c}\mathrm{A}_{V} \\
(\mathrm{mag})\end{array}$ \\
\hline V1 & $00: 19: 36.1$ & $+35: 02: 25.6$ & RRab & 0.558 & 55533.592 & 25.81 & 25.32 & 1.49 & 1.17 \\
\hline V2 & $00: 19: 36.0$ & $+35: 04: 17.5$ & RRab & 0.5856 & 55477.717 & 25.86 & 25.46 & 0.66 & 0.53 \\
\hline V3 & $00: 19: 32.4$ & $+35: 00: 35.3$ & $\mathrm{AC}$ & 1.306 & 55477.700 & 24.29 & 23.82 & 1.33 & 1.06 \\
\hline $\mathrm{V} 4^{a}$ & $00: 19: 38.9$ & $+35: 04: 47.2$ & RRab & 0.649 & 55532.600 & 25.89 & - & 0.68 & - \\
\hline V5 & $00: 19: 34.7$ & $+35: 05: 30.4$ & $\mathrm{AC}$ & 1.043 & 55476.140 & 24.46 & 23.94 & 0.66 & 0.61 \\
\hline V6 & $00: 19: 21.6$ & $+35: 01: 01.9$ & RRc & 0.3887 & 55532.730 & 25.79 & 25.31 & 0.53 & 0.42 \\
\hline $\mathrm{V} 7^{a}$ & $00: 19: 44.8$ & $+35: 02: 40.5$ & RRc & 0.3905 & 55532.682 & 25.96 & - & 0.35 & - \\
\hline V8 & $00: 19: 18.3$ & $+35: 02: 05.7$ & $\mathrm{AC}$ & 0.5873 & 55533.583 & 24.43 & 23.99 & 0.87 & 0.69 \\
\hline V9 & $00: 19: 26.3$ & $+34: 59: 21.7$ & RRab & 0.6159 & 55533.702 & 25.77 & 25.24 & 0.75 & 0.60 \\
\hline V10 & $00: 19: 41.8$ & $+35: 05: 20.8$ & RRab & 0.6054 & 55531.635 & 25.77 & 25.27 & 1.10 & 0.87 \\
\hline V11 & $00: 19: 19.1$ & $+34: 59: 38.8$ & RRab & 0.6194 & 55533.592 & 25.83 & 25.32 & 1.16 & 0.92 \\
\hline $\mathrm{V} 12^{a}$ & $00: 19: 44.1$ & $+35: 05: 53.5$ & RRab & 0.689 & 55532.700 & 25.62 & - & 0.67 & - \\
\hline V13 & $00: 19: 37.9$ & $+35: 07: 22.3$ & RRab & 0.5860 & 55477.735 & 25.86 & 25.42 & 0.98 & 0.78 \\
\hline V14 & $00: 19: 23.1$ & $+34: 57: 08.5$ & RRc & 0.4010 & 55531.653 & 25.69 & 25.26 & 0.56 & 0.45 \\
\hline V15 & $00: 19: 23.8$ & $+34: 56: 47.7$ & RRab & 0.5851 & 55532.790 & 25.80 & 25.27 & 0.77 & 0.61 \\
\hline V16 & 00:19:58.1 & $+35: 02: 59.6$ & RRc & 0.3748 & 55531.795 & 25.74 & 25.35 & 0.64 & 0.51 \\
\hline V17 & $00: 19: 58.4$ & $+35: 02: 57.6$ & RRab & 0.6105 & 55533.695 & 25.83 & 25.42 & 1.06 & 0.84 \\
\hline V18 & $00: 19: 04.7$ & $+35: 02: 58.3$ & RRab & 0.6167 & 55477.723 & 25.80 & 25.35 & 1.17 & 0.93 \\
\hline V19 & $00: 19: 55.0$ & $+34: 58: 25.6$ & $\mathrm{AC}$ & 1.2150 & 55533.830 & 24.26 & 23.87 & 1.54 & 1.22 \\
\hline V20 & 00:19:03.6 & $+35: 00: 32.5$ & $\mathrm{AC}$ & 1.146 & 55480.720 & 24.43 & 23.86 & 0.80 & 0.63 \\
\hline V21 & $00: 19: 29.6$ & $+34: 55: 12.2$ & RRab & 0.6052 & 55531.686 & 25.63 & 25.27 & 0.89 & 0.71 \\
\hline V22 & $00: 19: 55.5$ & $+35: 07: 30.1$ & RRc & 0.4090 & 55531.710 & 25.76 & 25.33 & 0.49 & 0.45 \\
\hline V23 & $00: 19: 29.8$ & $+34: 54: 39.8$ & RRc & 0.4076 & 55477.718 & 25.69 & 25.26 & 0.57 & 0.38 \\
\hline V24 & $00: 19: 30.5$ & $+34: 54: 29.2$ & $\mathrm{AC}$ & 0.5839 & 55533.785 & 24.90 & 24.51 & 1.28 & 1.02 \\
\hline V25 & $00: 20: 06.6$ & $+35: 03: 54.8$ & RRab & 0.643 & 55477.717 & 25.73 & 25.36 & 1.02 & 0.81 \\
\hline $\mathrm{V} 26^{a}$ & $00: 19: 33.3$ & $+35: 11: 27.7$ & RRab & 0.575 & 55532.767 & 25.75 & - & 1.02 & - \\
\hline V27 & $00: 19: 55.8$ & $+34: 56: 05.9$ & RRab & 0.6653 & 55532.735 & 25.57 & 25.17 & 1.06 & 0.85 \\
\hline V28 & $00: 19: 47.1$ & $+35: 10: 59.9$ & RRab & 0.6423 & 55531.742 & 25.88 & 25.34 & 1.07 & 0.85 \\
\hline $\mathrm{V} 29^{a}$ & $00: 20: 10.9$ & $+35: 04: 53.5$ & RRab & 0.6164 & 55531.780 & 25.74 & - & 0.81 & - \\
\hline V30 & $00: 18: 51.7$ & $+35: 04: 34.1$ & RRab & 0.6291 & 55477.106 & 25.73 & 25.25 & 1.21 & 0.96 \\
\hline V31 & $00: 20: 10.4$ & $+35: 07: 30.4$ & RRab & 0.6433 & 55533.793 & 25.82 & 25.42 & 0.85 & 0.69 \\
\hline V32 & $00: 18: 58.6$ & $+34: 55: 38.4$ & $\mathrm{AC}$ & 1.0513 & 55477.790 & 24.47 & 24.10 & 1.15 & 0.92 \\
\hline V33 & $00: 18: 59.7$ & $+35: 10: 21.1$ & RRab & 0.640 & 55533.733 & 26.15 & 25.65 & 1.11 & 0.88 \\
\hline V34 & $00: 20: 16.9$ & $+35: 02: 24.0$ & RRab & 0.6439 & 55531.670 & 25.83 & 25.35 & 0.66 & 0.52 \\
\hline V35 & $00: 19: 26.9$ & $+35: 14: 24.9$ & $\mathrm{RRc}$ & 0.330 & 55532.790 & 25.84 & 25.44 & 0.77 & 0.62 \\
\hline $\mathrm{V} 36^{a}$ & $00: 20: 03.2$ & $+35: 12: 33.9$ & $\mathrm{AC}$ & 0.6230 & 55532.650 & 24.55 & - & 0.48 & - \\
\hline V37 & $00: 18: 40.8$ & $+35: 05: 14.3$ & RRab & 0.6070 & 55531.669 & 25.82 & 25.30 & 1.50 & 1.19 \\
\hline V38 & $00: 18: 59.3$ & $+35: 12: 59.7$ & $\mathrm{RRc}$ & 0.4310 & 55533.810 & 25.59 & 25.21 & 0.33 & 0.32 \\
\hline V39 & $00: 20: 19.7$ & $+34: 52: 48.8$ & RRab & 0.5787 & 55532.750 & 25.64 & 25.31 & 0.75 & 0.60 \\
\hline
\end{tabular}

\section{Notes:}

${ }^{a}$ Only $B$ light curves are available for these stars, the $\langle\mathrm{B}\rangle$ values were obtained by adding to the instrumental $\langle\mathrm{b}\rangle$ values the zero point of the $B$ calibration equation. 
Table 3: $B, V$ photometry of the variable stars detected in And XIX. This Table is published in its entirety in the electronic edition of the Journal. A portion is shown here for guidance regarding its form and content.

\begin{tabular}{cccccc}
\hline \hline \multicolumn{7}{c}{ And XIX - Star V1 - RRab } \\
\hline $\begin{array}{c}\text { HJD } \\
(-2455000)\end{array}$ & $\begin{array}{c}\text { B } \\
(\mathrm{mag})\end{array}$ & $\begin{array}{c}\sigma_{B} \\
(\mathrm{mag})\end{array}$ & $\begin{array}{c}\text { HJD } \\
(-2455000)\end{array}$ & $\begin{array}{c}\mathrm{V} \\
(\mathrm{mag})\end{array}$ & $\begin{array}{c}\sigma_{V} \\
(\mathrm{mag})\end{array}$ \\
\hline 477.72208 & 26.03 & 0.30 & 531.66930 & 25.69 & 0.23 \\
531.65805 & 26.51 & 0.30 & 531.75167 & 25.85 & 0.18 \\
531.66338 & 26.52 & 0.24 & 531.79774 & 25.69 & 0.19 \\
531.66935 & 26.39 & 0.27 & 531.80311 & 25.65 & 0.27 \\
531.70489 & 26.56 & 0.32 & 533.58629 & 24.76 & 0.14 \\
531.71021 & 26.47 & 0.35 & 533.59162 & 24.81 & 0.13 \\
531.71570 & 26.34 & 0.24 & 533.59698 & 24.52 & 0.15 \\
531.75178 & 26.17 & 0.18 & 533.63227 & 25.02 & 0.08 \\
531.75713 & 26.24 & 0.18 & 533.63763 & 24.93 & 0.10 \\
531.76244 & 26.25 & 0.16 & 533.64302 & 24.90 & 0.07 \\
\hline
\end{tabular}

Magellanic Cloud, LMC, of 18.52 \pm 0.09 mag) and corrected for the different metal abundance using the relation $\frac{\Delta \mathrm{M}_{\mathrm{V}}}{\Delta[\mathrm{Fe} / \mathrm{HI}}=0.214 \pm 0.047 \mathrm{mag} / \mathrm{dex}$ by Clementini et al. (2003) and Gratton et al. (2004). We adopted for And XIX the metallicity $[\mathrm{Fe} / \mathrm{H}]=-1.8 \pm 0.3$ dex derived spectroscopically by Collins et al. (2013). The distance modulus of And XIX derived under the above assumptions is: $(\mathrm{m}-\mathrm{M})_{0}=24.66 \pm 0.17 \mathrm{mag}$. An independent reddening estimate can be obtained from the RR Lyrae stars using Piersimoni et al. (2002)'s method which is based on the relation between intrinsic $(B-V)_{0}$ color, period, metallicity and $B$-amplitude of the RRab stars. Using the 19 RRab stars for which we have photometry in both the $B$ and $V$ bands, we obtain $\mathrm{E}(\mathrm{B}-\mathrm{V})=0.11 \pm 0.06$ mag. The distance modulus derived with this new reddening is: $(\mathrm{m}-\mathrm{M})_{0}=24.52 \pm 0.23$ mag. Both our distance moduli place And XIX almost at the same distance of M31 and both are in good agreement, within the errors, with the value of $(\mathrm{m}-\mathrm{M})_{0}=24.57_{-0.43}^{+0.08} \mathrm{mag}$ found by Conn et al. (2012). Our estimates are instead smaller than McConnachie et al. (2008) modulus for And XIX, but still consistent with their value within $1 \sigma$.

\section{THE CMD}

Figure 4 shows the CMDs obtained in the present study selecting objects in different regions of the FoV.
To avoid contamination from background galaxies and peculiar objects, we selected our photometric catalog using the $\chi$ and Sharpness parameters provided by ALLFRAME. We only retained sources for which $-0.3 \leq$ Sharpness $\leq 0.3$ and with $\chi<1.0$ for magnitudes fainter than $V=$ $22.0 \mathrm{mag}$, and $\chi<1.5$ for magnitudes brighter than $V=22.0 \mathrm{mag}$. These selections led to a total of $\sim 9000$ stars, plotted as dots in Fig. 4 The variable stars are plotted in Fig. 4 according to their intensity-average magnitudes and colors and using red circles for the RRab stars, blue triangles for the RRc stars, and green squares for the ACs. Only 26 RR Lyrae stars and 7 ACs could be plotted, as we lack $V$ photometry for $5 \mathrm{RR}$ Lyrae stars and 1 AC. The right panel of Figure 4 shows the CMD of the whole FoV of the LBC observations; the $1 \sigma$ error bars, as derived from artificial star tests conducted on real images, are also drawn. The left panel shows only stars inside the ellipse drawn with the galaxy half-light radius $\left(\mathrm{r}_{h}=6.2^{\prime}\right.$, McConnachie et al. 2008; see Fig. 2), and the central panel shows the CMD of stars inside an elliptical ring with internal radius $6.2^{\prime}$ and external radius $8.8^{\prime}$, which encloses the same area as in the left panel. The most prominent features of the And XIX CMD are:

- a RGB, between $B-V=0.6-1.5 \mathrm{mag}$, extending upwards to $V \approx 22-22.5 \mathrm{mag}$;

- a red HB with colors $0.6<B-V<0.8$ mag; 


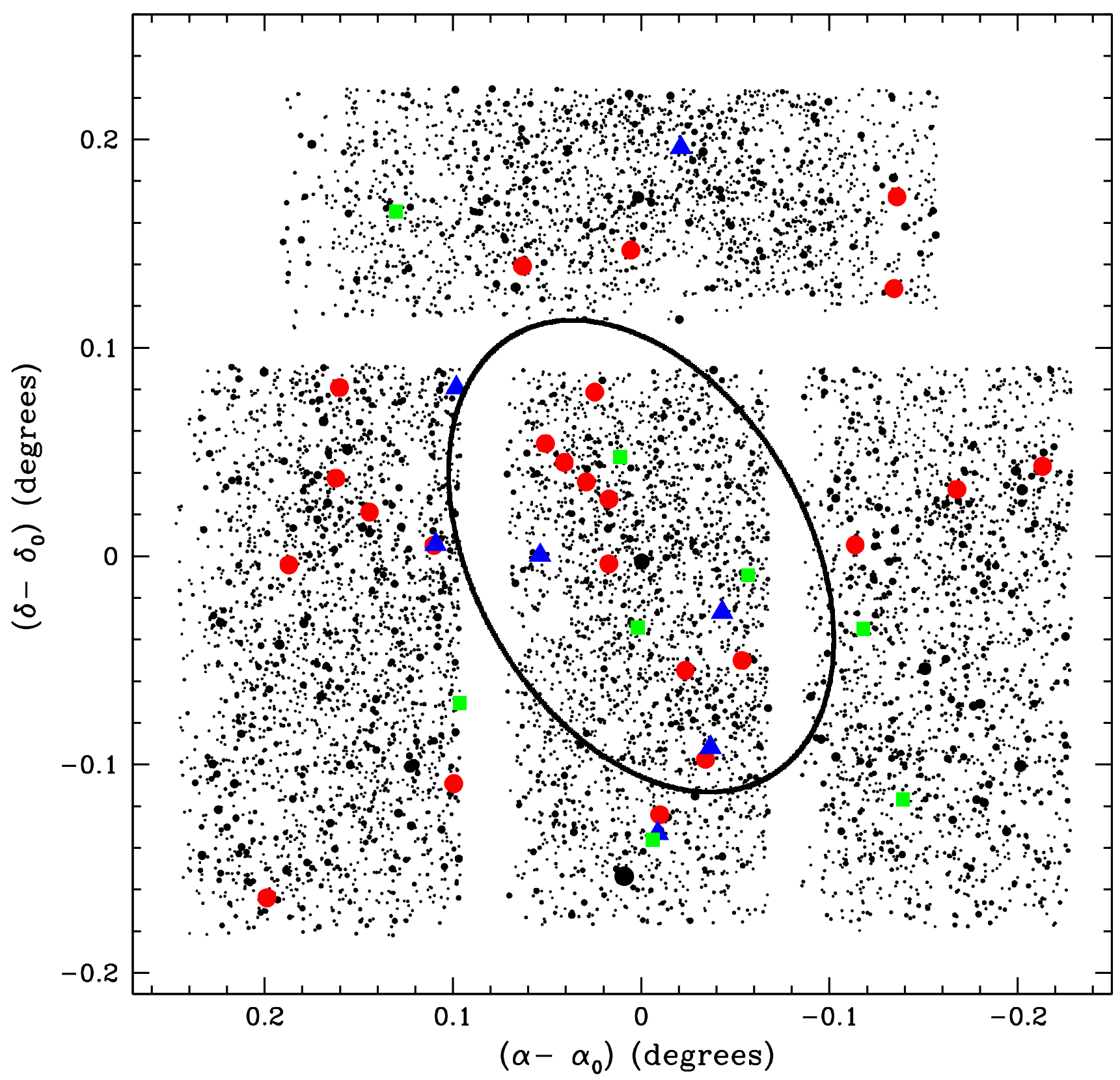

Fig. 2.- Spatial distribution of the variable stars identified in the FoV covered by our LBC observations of And XIX. Red circles and blue triangles mark RRab and RRc stars, respectively. Green squares are ACs. Black dots are non-variable stars: they are plotted with symbol sizes inversely proportional to their magnitude. The black ellipse is drawn by convolving And XIX half-light radius with the galaxy ellipticity and position-angle following McConnachie et al. (2008) . 


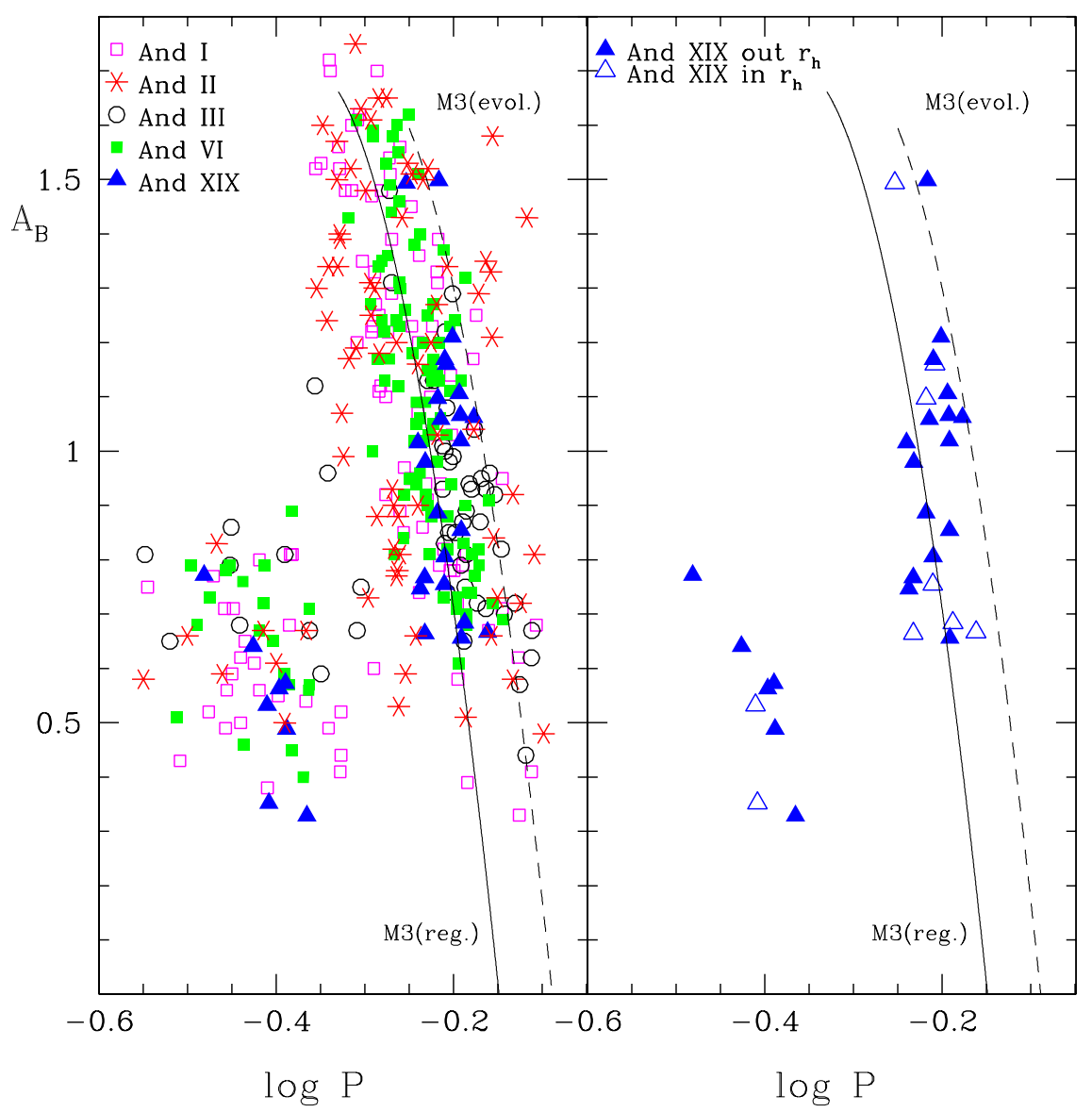

Fig. 3.- Right: Period-amplitude diagram in the $B$-band for the RR Lyrae stars identified in the present study. Open symbols mark variables falling inside the ellipse shown in Fig. 2 Also shown, for comparison, are the loci defined by the bona-fide regular (solid curve) and well-evolved (dashed curve) fundamental-mode RR Lyrae stars in M3, from Cacciari et al. (2005); Left: comparison of And XIX variables with the RR Lyrae stars stars identified in other four M31 dwarf satellites (namely, And I, And II, And III and And VI).

- a distribution of sources extending nearly vertically at $B-V \sim 0.2-0.4$ mag; the unusual color of these objects (see Sect 9), can be explained by unresolved background galaxies;

- a sequence of stars at $B-V \sim 1.5-1.7$ mag and extending blueward around $V=$ $22 \mathrm{mag}$. This feature is likely composed by foreground field stars (also see Section 9).

In the left and center panels of Fig. 4 we have plotted in blue the ridge lines of the Galactic globular cluster NGC 5824 from Piotto et al.
(2002), corrected to the distance and reddening of And XIX we derived from the RR Lyrae stars (see Sect. 5). We assumed for NGC 5824 a distance modulus of $(\mathrm{m}-\mathrm{M})_{0}=17.54 \mathrm{mag}$ and a reddening of $\mathrm{E}(\mathrm{B}-\mathrm{V})=0.13 \mathrm{mag}$ from Harris (1996) cata$\log$ (2010 edition). NGC 5824 has a metallicity $[\mathrm{Fe} / \mathrm{H}]=-1.94 \pm 0.14$ dex (Carretta et al. 2009) that is very similar to the metal abundance of And XIX estimated by Collins et al. (2013). The RGB of NGC 5824 matches very well And XIX's RGB thus confirming both the similar metallicity and the higher reddening value inferred from the RR Lyrae stars. The IS boundaries for RR Lyrae 
stars and ACs with $Z=0.0002$ from Marconi et al. (2004) are overplotted to the CMD in the right panel of Fig. 4. The variables we have classified as RR Lyrae stars fall well inside the boundaries of the RR Lyrae IS, confirming they are bona-fide RR Lyrae stars. Similarly, the variables above the HB appear to be confined in the region of the CMD where ACs are usually found (see following section).

\section{ANOMALOUS CEPHEIDS}

In And XIX we have identified 8 variable stars from about 1 to 1.5 mag brighter than the average $B$ magnitude of the HB. These stars are found to fall inside the boundaries of the IS for ACs from Marconi et al. (2004) (see Fig. 4), thus providing support to their classification as ACs. In order to investigate further their nature we have also compared them with the $P L$ relations for ACs. ACs follow a Period-Luminosity $(P L)$ relation which differs from both the Classical Cepheids and the type II Cepheids $P L$ relationships (see Fig. 1 of Soszvnski et al. 2008a). Unfortunately, the $P L$ relation has the disadvantage of being reddening dependent and in some cases the scatter around the mean value can be very high. Narrower relations are found introducing a color term in the $P L$ relation and in particular the Wesenheit function (van den Bergh 1975; Madore 1982) includes a color term whose coefficient is equal to the ratio between total-to-selective extinction in a filter pair. In such a way the $P W$ relation is reddening free by definition. The Wesenheit index in our case is $\mathrm{W}(B, V)=\mathrm{M}_{V}-3.1 \times(B-V)$, where $\mathrm{M}_{V}$ is the $V$ magnitude corrected for the distance. We have $B$ and $V$ magnitudes for 7 of the variables above the HB. Their $\langle V\rangle$ magnitudes were corrected using the distance modulus $(\mathrm{m}-\mathrm{M})_{0}=24.52$ mag derived from the RR Lyrae stars and used to derive the corresponding Wesenheit indices. The position of these 7 variables in the $P W$ plane is shown in the left panel of Figure 5 where we also plot, as solid lines, the $P W$ relations for ACs recently derived by Ripepi et al. (2013) using 25 ACs in the LMC3 (LMC). Six of And XIX bright variables appear to fall well on the Ripepi et al.

[Ripepi et al. (2013)'s relations were derived for the $V$ and $I$ bands, and we have converted them to $B$ and $V$ using Equation 12 of Marconi et al. (2004). (2013)'s $P W$ relations for ACs with stars V3, V19, V32 likely being fundamental-mode pulsators, and stars V5, V20, V24 likely pulsating in the firstovertone mode. On the other hand, star V8 appears to be more than $2 \sigma$ off the first-overtone $P W$ relation, hence its classification as $\mathrm{AC}$ seems to be less robust. To show that these bright variables are mostly ACs, on the right panel of Figure 5 the $P W$ relation for Classical Cepheids (CCs) in the LMC derived by Soszynski et al. (2008b) is shown. The $P W$ relation for CCs indeed does not fit very well the bright variables in And XIX.

We will further discuss the nature of the bright variables of And XIX in Sect. 10.2 where we compare them with theoretical isochrones.

Mateo et al. (1995) found that the specific frequency of ACs (i.e. the number of ACs per $10^{5}$ $\mathrm{L}_{V}$ ) in the Galactic dSph galaxies is related to the luminosity and metallicity of the parent $\mathrm{dSph}$. Pritzl et al. (2004, 2005) found that this correlation also holds for the M31's satellites And I, And II, And III and And VI. On the assumption that the 8 supra-HB variables of And XIX are ACs, in Figure 6 we plot the specific frequency of ACs in And XIX versus its luminosity (left panel) and metallicity (right panel) and compare it to the one in Galactic and M31 dSphs from Pritzl et al. (2004). And XIX follows well the relation traced by the other dSphs. This also indicates that we have detected almost all the short period variable stars at $V \sim 24 \mathrm{mag}$ in And XIX, as also dictated by the artificial star test that gives a completeness of $\sim 80 \%$ at this level of magnitude.

\section{M31 HALO CONTAMINATION}

Although And XIX is far $(\sim 120 \mathrm{kpc}$, Conn et al. 2012) from the M31 center the contamination from RR Lyrae stars and ACs belonging to the M31 halo may be not negligible. The FoV of the LBC $\left(\sim 0.15 \mathrm{deg}^{2}\right)$ is such that we were able to fit in just one pointing a large portion of And XIX $\left(\simeq 2 \times r_{h}\right)$. Unfortunately, in such a large FoV contaminants are also expected to be present in a large number. As we showed in Sect. [5 And XIX is almost at the same distance of M31 and for this reason the luminosity of the variable stars in And XIX and in the M31 halo is similar, thus distinguishing the two samples on the basis of the average luminosity is not possible. To estimate 


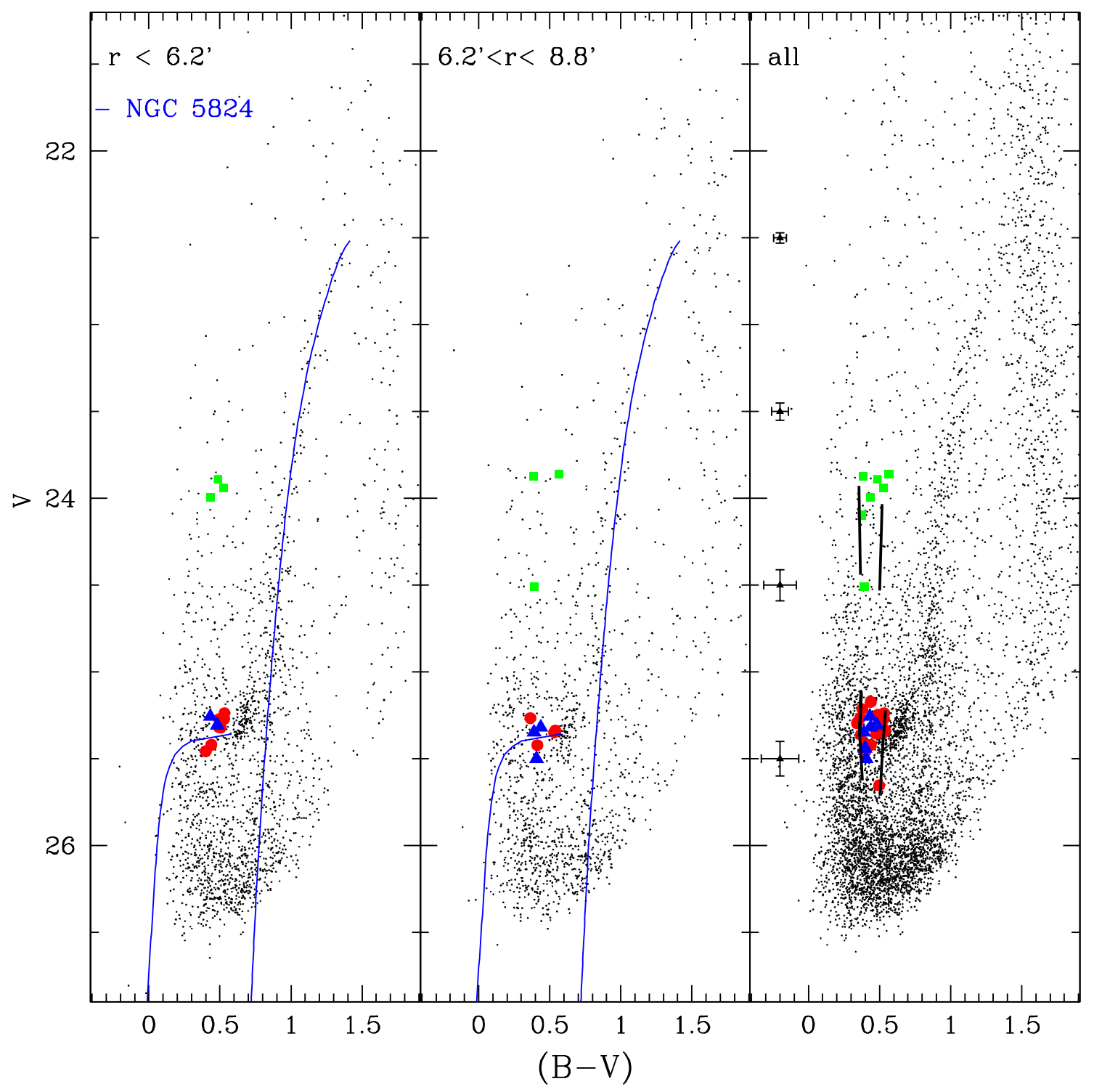

Fig. 4.- CMDs obtained selecting objects in different regions of the FoV. Only the sources for which $-0.3 \leq$ Sharpness $\leq 0.3$ and $\chi<1.0$ for magnitudes fainter than $V=22.0$ mag, and $\chi<1.5$ for magnitudes brighter than $V \sim 22.0 \mathrm{mag}$, are displayed in the three panels of the figure, in order to reduce contamination from background galaxies and peculiar objects. Red circles are RRab stars, blue triangles are RRc stars, and green squares are ACs. Left: CMD of only stars inside an ellipse drawn with the galaxy half-light radius (see Fig. 2). Shown in blue are the ridgelines of the Galactic globular cluster NGC5824 $([\mathrm{Fe} / \mathrm{H}]=-1.94$ dex, Carretta et al. 2009); Middle: same as in the left panel, but for stars inside an elliptical ring with internal radius $6.2^{\prime}$ and external radius $8.8^{\prime}$, which encloses the same area as in the left panel; Right: CMD of stars in the whole LBC FoV. Black solid lines show the boundaries of the IS for RR Lyrae stars and ACs with $Z=0.0002$, from Marconi et al. (2004). 


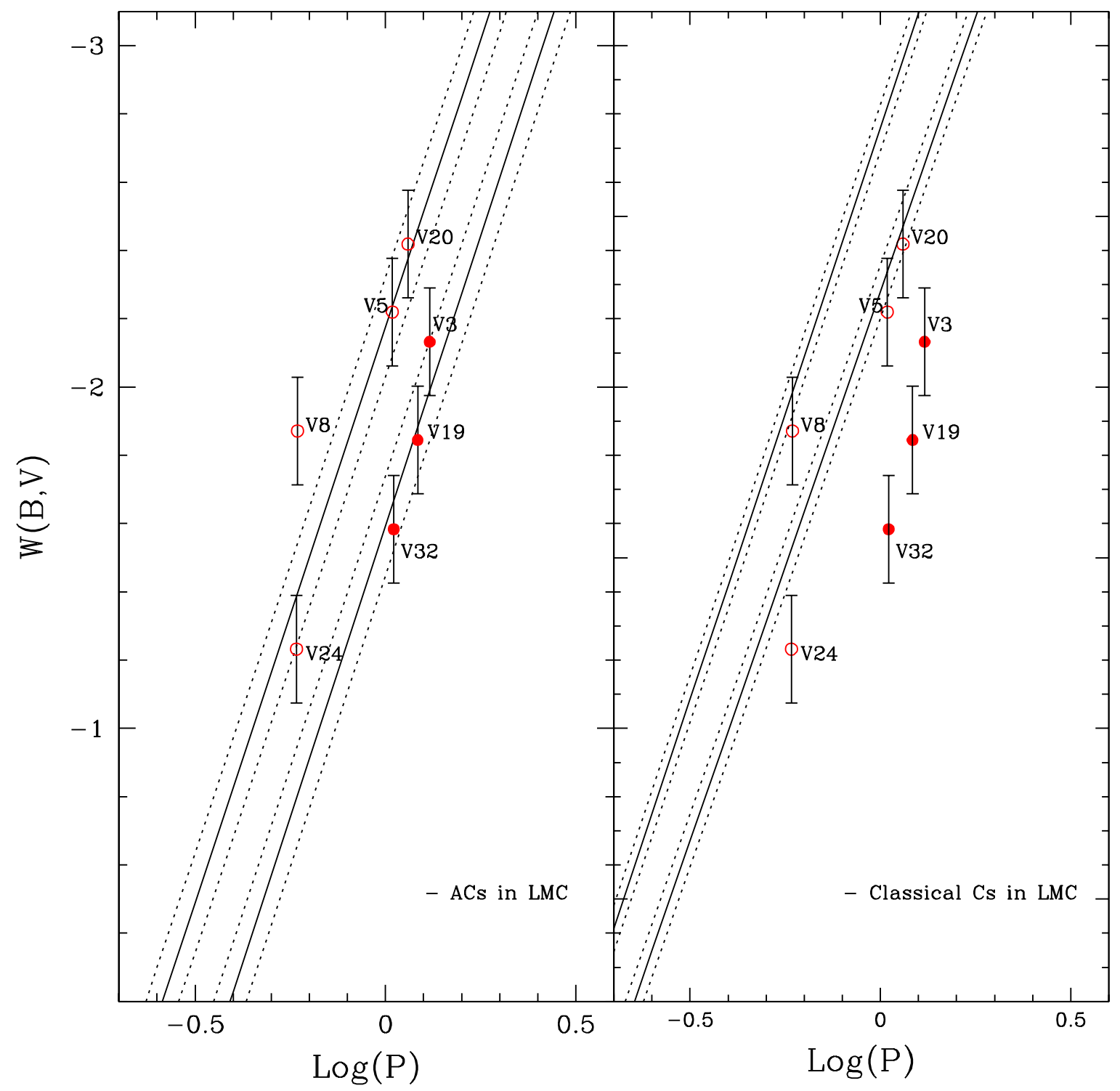

Fig. 5.- Left panel: $P W$ relations for And XIX's variables brighter than the HB. Solid lines are the fundamental-mode (lower line) and the first-overtone (upper line) $P W$ relations for ACs by Ripepi et al. (2013) (converted to $B, V$ bands using the relations in Marconi et al. 2004), with their related $1 \sigma$ uncertainties. Right panel: same as left panel, but with the $P W$ relations for Classical Cepheids by Soszynski et al. (2008b)

how many variable stars belonging to the M31 halo can be expected to contaminate And XIX's sample we used the results from the search for variable stars in 6 fields around M31 made by
Jeffery et al. (2011) using HST/ACS. Two of these fields are in the halo of M31 at a distance of about $35 \mathrm{kpc}$ from the center in the south-east direction along the galaxy minor axis. They were found 


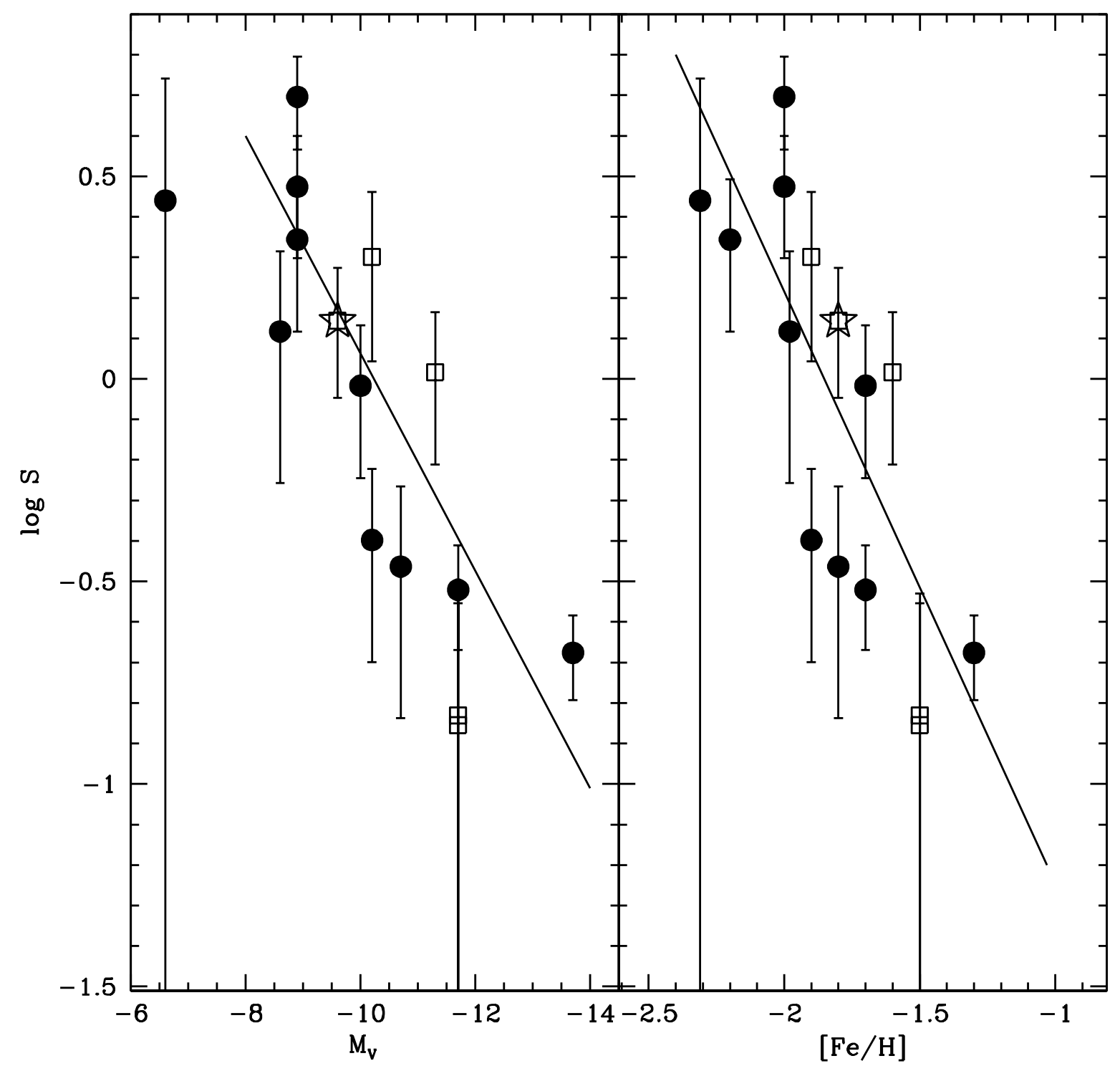

Fig. 6.- Specific frequency of ACs in MW (filled circles) and M31 (open squares) dSphs vs. absolute visual magnitude $\left(\mathrm{M}_{\mathrm{V}}\right.$, left panel $)$, and metallicity $([\mathrm{Fe} / \mathrm{H}]$, right panel $)$ of the parent galaxy. And XIX is marked by a star symbol.

to contain 5 and no RR Lyrae stars, respectively, despite they have comparable stellar density. Furthemore, none of the two halo fields was found to contain ACs. From these results we can give a rough estimate of how many RR Lyrae stars and ACs belonging to M31 we expect to find in the field of And XIX. Assuming a stellar density of the M31 halo of $\propto \mathrm{r}^{-3.2}$ (Gilbert et al. 2012) and after scaling for the different area surveyed by the LBC and HST/ACS we estimated a contamination from the M31 halo by 5 RR Lyrae and no ACs. Even if these could be underestimates, 
the number of possible contaminants from M31 appears to be in any case small compared to the 31 RR Lyrae stars and 8 ACs we have found in And XIX. On the basis of the arguments above, we conclude that the contamination from M31 halo does not affect significantly our results.

\section{SPATIAL DISTRIBUTION}

In order to explore the spatial structure of And XIX, Figure 7 shows the spatial distributions of stars from different regions of the CMD (Fig. 8). To better visualize the maps, the data points were binned to a pixel size of 7 arcsec and smoothed with a 2D Gaussian kernel with $\sigma=20$ arcsec. In Figure 7 from the top left panel clockwise we show MW (green symbols in Fig. 8) stars, And XIX RGB and HB stars (magenta symbols in Fig. 8), intermediate color objects (blue symbols in Fig. 8) and blue objects (cyan symbols in Fig. 8). Variable stars (red circles for RR Lyrae stars, and green squares for ACs) are also overlaid to the density maps.

As expected, the MW stars are homogeneously distributed all over the field. On the other hand, RGB and HB stars seem to be concentrated along a diagonal bar-like structure running from southwest to northeast and pointing toward the M31 center. Along this bar are also positioned 20 of the 39 variable stars. Interestingly, the distribution of the blue objects does not correlate with the distribution of RGB and HB stars, which are clearly members of And XIX, but shows an overdensity in the upper CCD of the LBC camera, in a region around R.A. $=4.84^{\circ}$, dec $=+35.22^{\circ}$, and radius $\sim 2.5$ arcmin. We suggest that these blue objects are most likely unresolved galaxies. To examine in depth this possibility we calculated an upper limit for the number of unresolved galaxies expected in the FoV of our LBC observations using the HST Ultra Deep Field (UDF) catalog of galaxies by Coe et al. (2006) and making the assumption that the distribution of galaxies in the sky is almost isotropic. In the UDF catalog we selected galaxies with the same range of colors and magnitudes used to select the blue objects in our CMD. Furthermore, we selected galaxies with radii smaller than 0.75 arcsec, which, given the average seeing of the LBC images, should result in unresolved objects. After scaling for the different area surveyed by the UDF catalog of Coe et al. (2006) and by the LBC, we end up with an estimated upper limit of 1450 unresolved galaxies in the LBC field. The number of blue objects in the LBC catalog is 1736 that is comparable to the upper limit found from the UDF catalog. This seems to support our claim that the majority of the blue objects in the CMD are likely unresolved galaxies. As a further evidence Fig. 9 shows isochrones with solar metallicities overlaid to the CMD. This figure shows that the blue sources in the CMD (in cyan in Fig. (8) are much redder than solar isochrones, so more consistent with unresolved galaxies than single stars. In this case the overdensity in the upper CCD is likely a cluster of galaxies. We searched the Wen \& Han (2013) catalog for known clusters of galaxies around the center of the overdensity $\left(\sim\right.$ R.A. $\left.=4.84^{\circ}, \operatorname{dec}=35.22^{\circ}\right)$, but we did not find any in a radius of $\sim 2.5$ arcmin. However, we notice that Wen \& Han (2013) catalog is based on SDSS data, that are shallower when compared to our deep photometry.

Finally, the distribution of intermediate color objects partially overlaps with the RGB/HB distribution (which is peaked in the central CCD), but shows mild overdensities in the upper and right hand CCDs as well. This sample is likely mostly populated by unresolved galaxies, although there are probably also some members of And XIX. Indeed the ACs are in this region of the CMD, and are contributing to these overdensities. However, only high resolution observations with HST will allow a better galaxies/stars separation, thus helping in clarifying whether And XIX hosts an intermediate/young age component.

\section{CMD INTERPRETATION}

\subsection{Old population: two episodes of star formation}

Overlaid to the CMD in Figure 10 are shown the Padova isochrones of different ages and metallicities obtained using the CMD 2.5 web inferface4 based on models from Bressan et al. (2012). The adopted foreground reddening and the distance modulus are $\mathrm{E}(B-V)=0.11 \mathrm{mag}$ and $(m-M)_{0}=$ $24.52 \mathrm{mag}$, respectively, as derived from the RR Lyrae stars. Although the age-metallicity degen-

$\sqrt[4]{h t t p: / / s t e v . o a p d . i n a f . i t / c g i-b i n / c m d}$ 

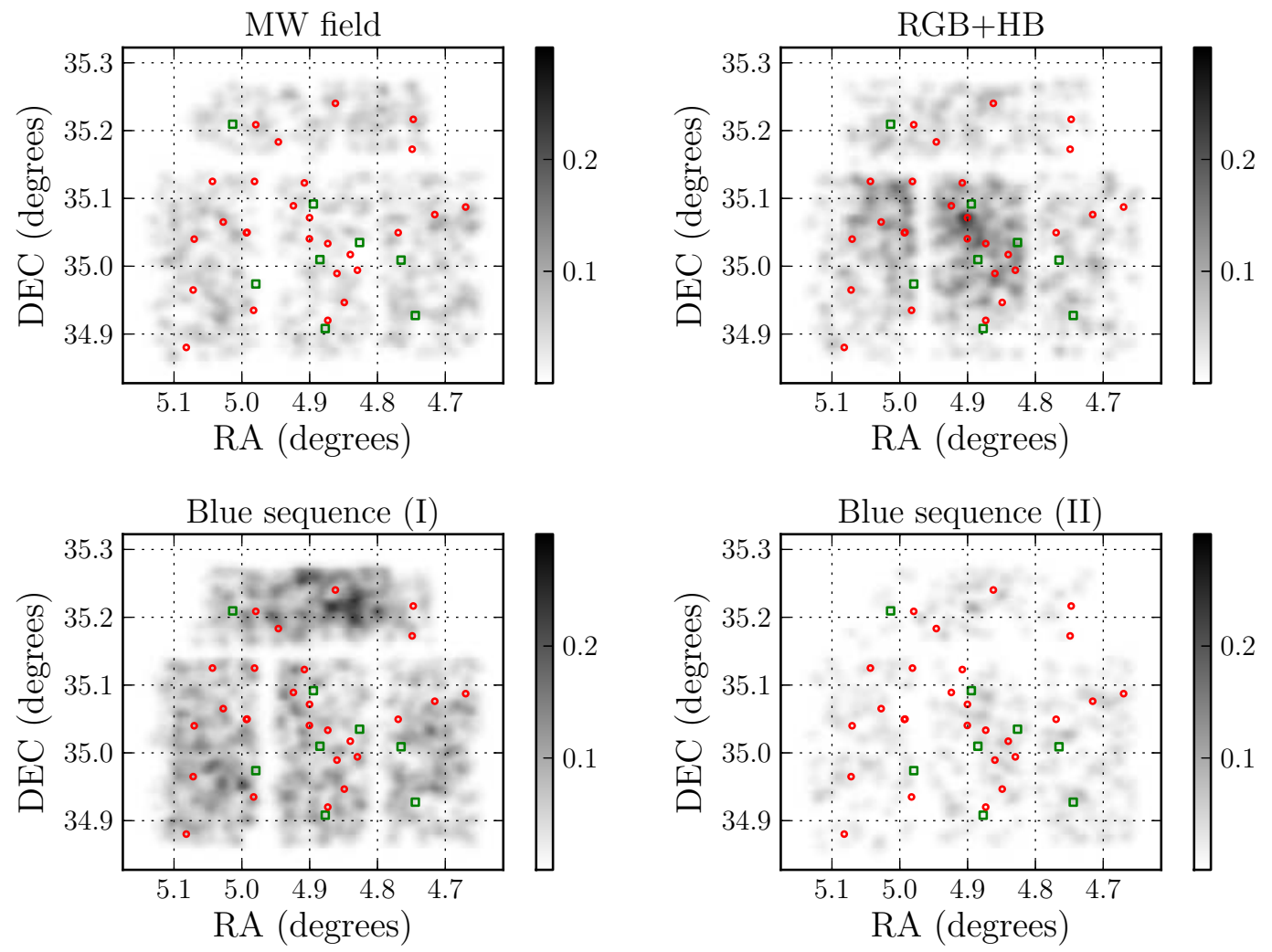

Fig. 7.- Density-contours of the objects selected using the CMD in Figure 8. From the upper left panel clockwise MW stars (in green in Figure 8), RGB +HB stars (in magenta in Figure 8), intermediate blue sequence objects (in blue in Figure 8) and blue sequence objects (in cyan in Figure 8). Red circles are RR Lyrae stars while green squares are ACs. 


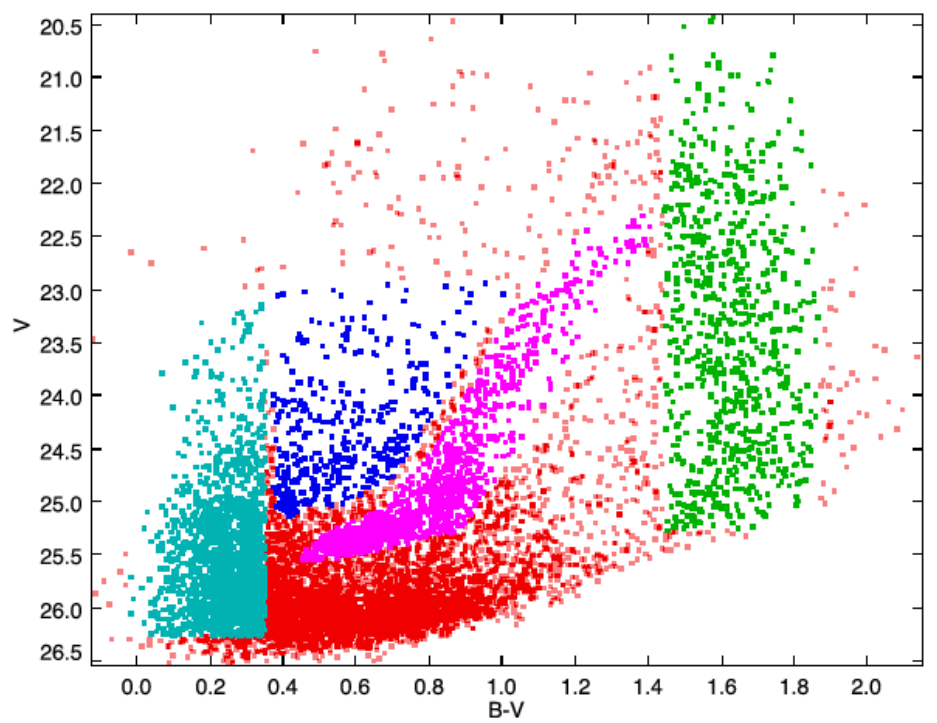

Fig. 8. - CMD of the sources in the FoV of our observations of And XIX satisfying the $\chi$ and Sharpness conditions described in Sect. 6 (red points), with marked in different colours the selections used to construct the spatial maps of Figure 7 RGB + HB stars (magenta), MW stars (green), blue objects (cyan) and intermediate blue objects (blue).

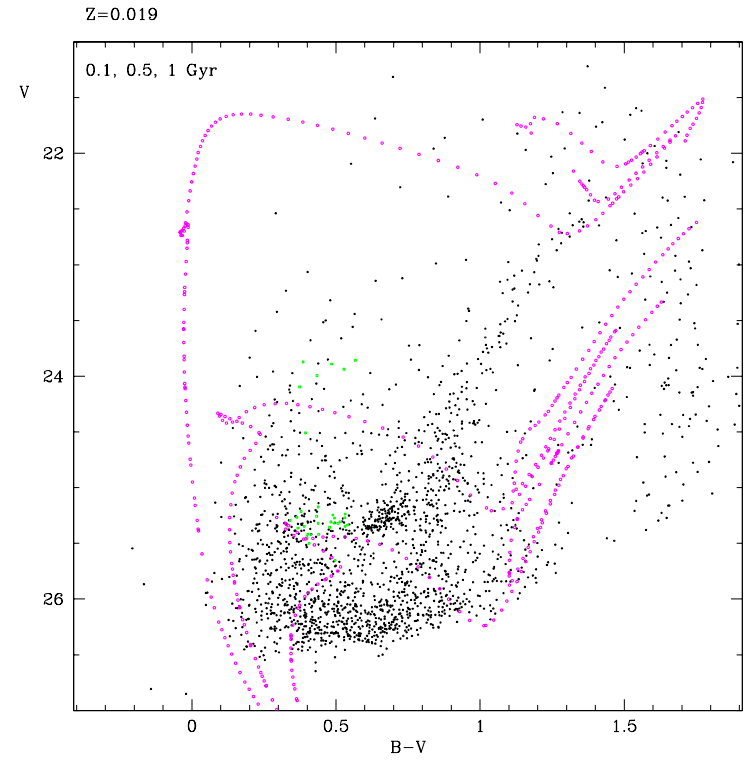

Fig. 9.- CMD with overlaid solar metallicity isochrones from 0.1 to 1 Gyr. eracy makes the interpretation of the RGB color troublesome, we can still find feasible scenarios by fitting the RGB and HB simultaneously. Our findings suggest that And XIX hosts two distinct stellar populations:

1. An old and metal poor component, hereinafter P1, as suggested by the presence of RR Lyrae stars. Our fit suggests that only metallicity $Z=0.0003\left(\log Z / Z_{\odot}=-1.80\right)$ and ages between 12 and 13 Gyr can fully match the location of the RGB and the average color of the RR Lyrae stars. Formally, we can rule out younger ages (the top right panel illustrates a 11 Gyr isochrone), since the corresponding RGB and $\mathrm{HB}$ are too blue and too red respectively, and higher metallicities $\left(Z=0.0004, \log Z / Z_{\odot}=-1.67\right.$; middle panels), since the predicted HB is clearly redder than the RR Lyrae color.

2. A more metal rich and possibly younger component, hereafter $\mathrm{P} 2$, as traced by the red HB. Based on the isochrone fitting, as shown in middle and bottom panels of Fig- 
ure 10, we find that isochrones in the metallicity range $Z=0.0004-0.0006\left(\log Z / Z_{\odot}=\right.$ $-1.67 /-1.5)$, with ages spanning from 6 to $10 \mathrm{Gyr}$, match well both the color extension of the red $\mathrm{HB}$ and the mean position of the RGB. However, the lowest metallicity isochrones actually fit best, while the more metal rich isochrones predict giants that are too red. Lowering the age of the isochrone $(<6$ Gyr) partially counters this effect, but also produces a too bright HB. Vice versa, isochrones older than $10 \mathrm{Gyr}$ at $Z=0.0004$, although producing tolerably good fits in the $\mathrm{RGB}$ region, miss the red $\mathrm{HB}$.

Unfortunately, the strong galactic contamination makes it difficult to quantify the fraction of blue HB stars. Likewise, the ratio between P1 and P2's star formation rates is very uncertain.

\subsection{A recent episode of star formation?}

The presence of pulsating stars brighter than RR Lyrae stars rises the question of whether And XIX has been forming stars up to 1 Gyr ago. In Figure 11 and 12 we show stellar evolutionary tracks from the Basti web-site 5 based on models by Pietrinferni et al. (2004) for metallicities $Z=$ 0.0003 and $Z=0.0006$ respectively, and masses in the range $0.8-2.4 \mathrm{M}_{\odot}$, overlaid on the observed CMD. We used the Basti tracks for this comparison because the $Z=0.0003$ and $Z=0.0006$ metallicities are not available for the Padova evolutionary tracks. In the $Z=0.0003$ case, the location of the pulsating stars is bracketed by 1.8 and 2.0 $M_{\odot}$ tracks, while in the $Z=0.0006$ case it is constrained by 2.0 and $2.2 M_{\odot}$ tracks. In terms of age, the best fitting isochrones (see Figure 13) suggest a range of ages 1-1.25 Gyr old at $Z=0.0003$ and $0.75-1$ Gyr old at $Z=0.0006$. Although this is not a large difference, we note that in the former scenario the pulsating stars are consistent with being ACs, as suggested by the RGB tip of similar luminosity for both the 1.8 and $2.0 M_{\odot}$ tracks (both masses are lower than the RGB transition mass), while in the latter they are at the borderline between being ACs and Short-Period Classical Cepheids, as suggested by the short RGB of the $2.2 M_{\odot}$ track compared to the $2.0 M_{\odot}$ track (the

$\sqrt[5]{h t t p: / / a l b i o n e . o a-t e r a m o . i n a f . i t / \mid}$
$2.2 M_{\odot}$ is above the RGB transition mass, the $2.0 M_{\odot}$ is below). Furthemore, the stellar evolutionary tracks predict the existence of ACs only for metallicities lower than $Z=0.0004$ (see, e.g., Marconi et al. 2004).

To further investigate the presence of young stars, two synthetic populations (see Cignoni \& Tosi 2010, for an overview of the technique) were generated following the two most likely scenarios, namely a metallicity $\mathrm{Z}=0.0006$ and a constant $\mathrm{SF}$ in the range 1-0.75 Gyr, and a metallicity $\mathrm{Z}=0.0003$ and a constant $\mathrm{SF}$ in the range 1.25 1.00 Gyr. For both models we used the Padova tracks (Marigo et al. 2008; Bertelli et al. 2009), convolved with photometric errors and incompleteness as estimated from artificial star tests, and a Salpeter initial mass function (IMF). Once the number of synthetic objects populating the region $23.5<V<24.5 \mathrm{mag}$ and $0.2<B-V<0.9$ mag equals the number of brighter pulsators, the procedure was stopped, giving the minimum amount of star formation necessary to generate the brighter variables. This led to a star formation rate of the order of $10^{-5} M_{\odot}$ per yr. Figure 14 shows the resulting simulations (blue pentagons and red triangles indicate $\mathrm{Z}=0.0006$ and $\mathrm{Z}=0.0003$ simulations, respectively) overlaid to the observed CMD. Although the exact CMD morphology of the ACs is not perfectly reproduced, both synthetic populations show a number of MS stars (objects at $V>25.5 \mathrm{mag}$ ) which is not much lower than star counts observed in the corresponding CMD regions. This suggests that, if ACs are associated with a genuine SF episode, our inferred rate can be considered an upper limit to the recent activity in And XIX.

The other way of accommodating the apparent youth is that these stars are the evolved counterpart of MS blue stragglers. In this case they are not the result of a recent episode of star formation, but rather the result of mass transfer in close binary systems occurred about 1 Gyr ago. Unfortunately, the detection of MS blue stragglers or genuine young stars in MS is greatly hindered by the contamination of blue galaxies.

\section{SUMMARY AND CONCLUSIONS}

We have presented $B$ and $V$ time-series observations of the M31 dSph satellite And XIX, that 


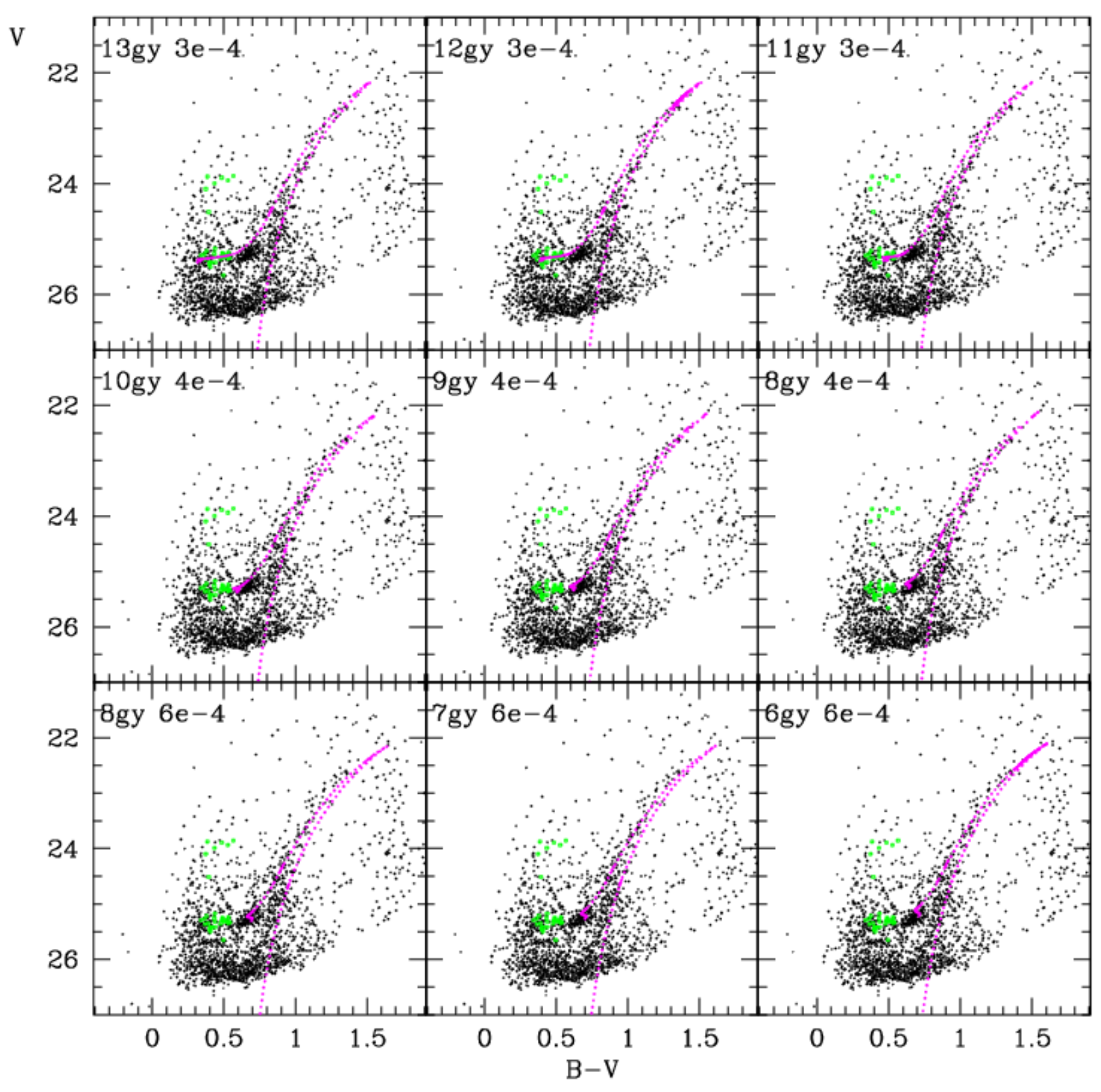

Fig. 10.- Observed CMD with overlaid stellar isochrones from the Padova evolutionary models on the CMD 2.5 web interface. Metallicities from the top to the bottom panel are $\mathrm{Z}=0.0003,0.0004$ and 0.0006 , respectively. The ages of the isochrones are indicated in each individual panel. Variable stars are represented by green squares. 


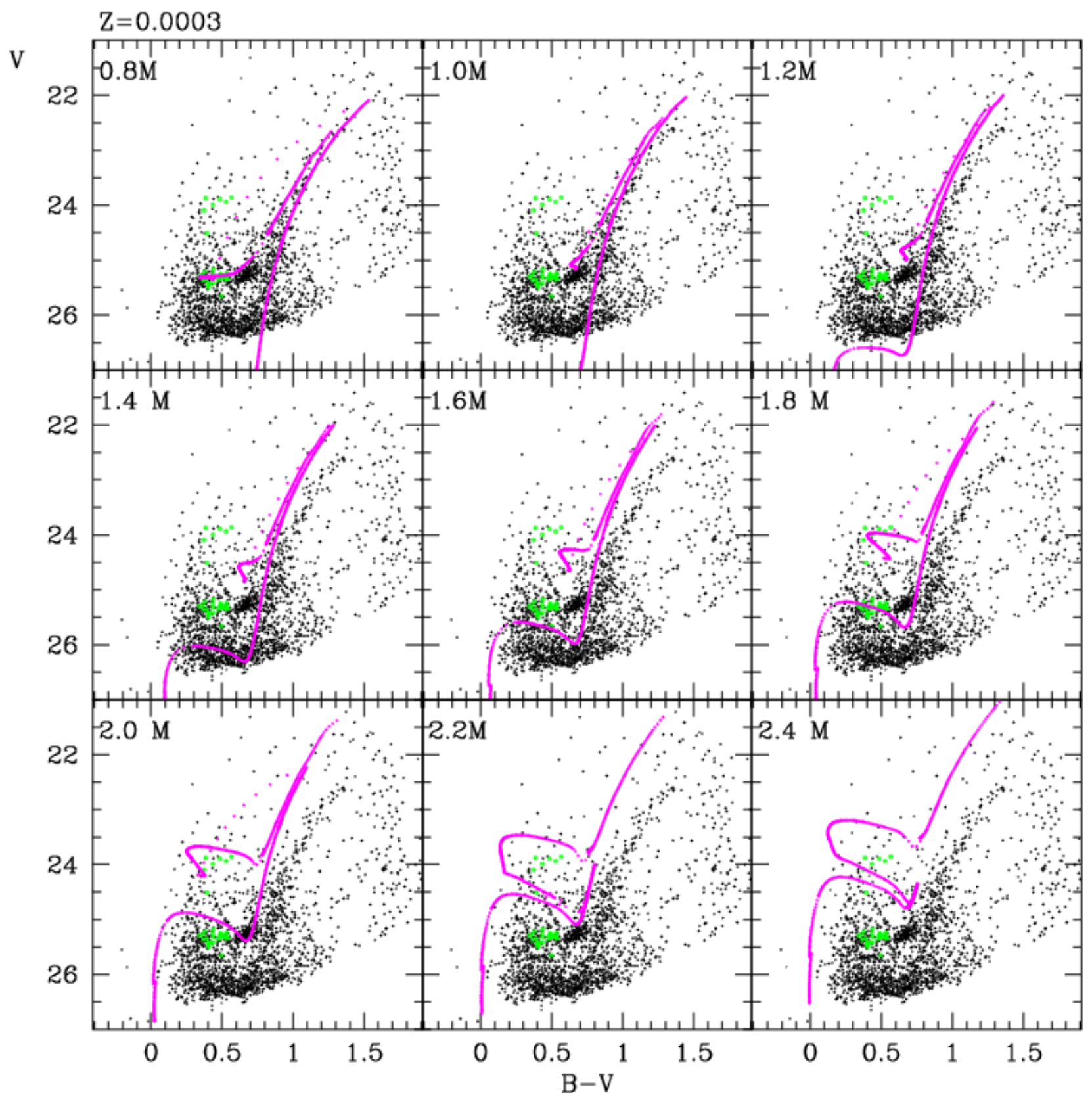

Fig. 11.- Stellar evolutionary tracks from the Basti web site. Starting from the top-left to the bottom-right masses from 0.8 to $2.4 \mathrm{M}_{\odot}$. The metallicity is $Z=0.0003$. Variable stars are represented with green squares.

we performed using the LBC at the LBT. A total number of 39 variable stars were identified in the galaxy of which 31 are RR Lyrae stars and 8 likely are ACs. From the average period of the 


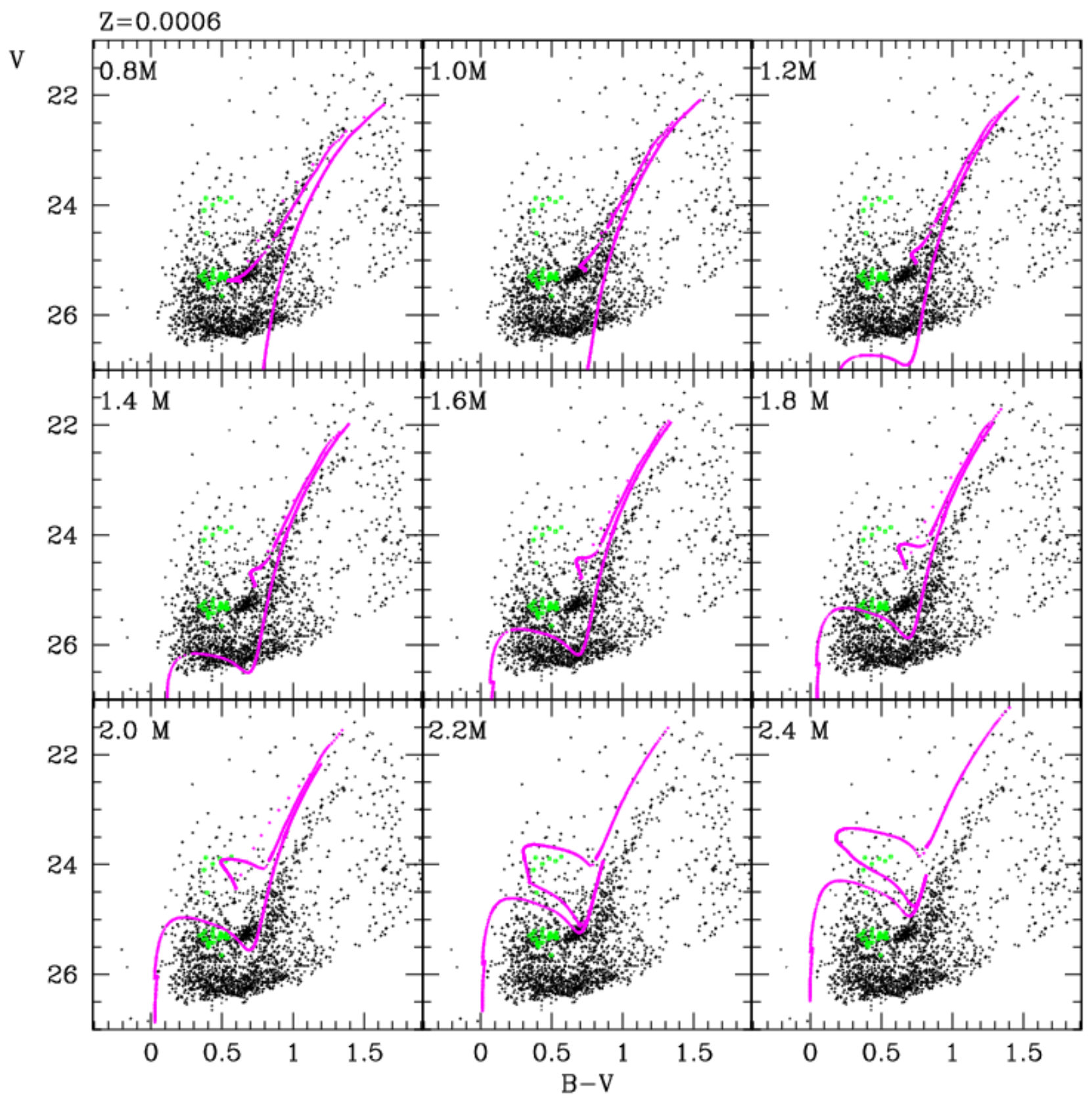

Fig. 12.- Same as Figure. 11, but for metallicity $Z=0.0006$.

RRab stars and the period-amplitude diagram we classify And XIX as an Oo-Int system. The average $V$ magnitude of the RR Lyrae stars allowed us to estimate the distance modulus of And XIX, $(\mathrm{m}-\mathrm{M})_{0}=24.52 \pm 0.23 \mathrm{mag}($ for $\mathrm{E}(\mathrm{B}-\mathrm{V})=0.11 \pm 0.06$ mag, as we derive from the RR Lyrae stars) or (m- 


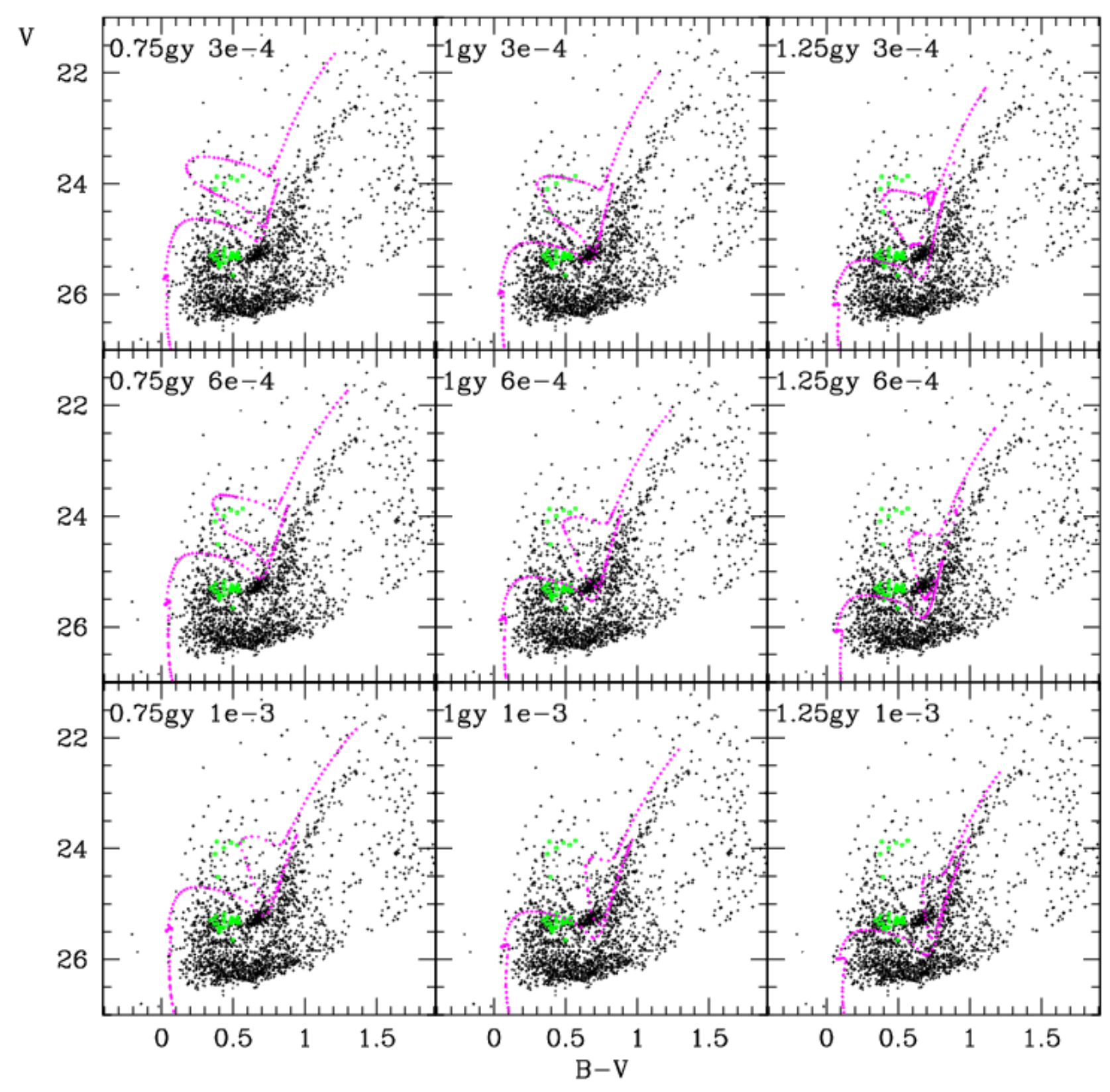

Fig. 13. - Same as Figure 10, but for metallicities from the top to the bottom panel of $\mathrm{Z}=0.0003,0.0006$ and 0.001 , respectively and younger isochrones.

$\mathrm{M})_{0}=24.66 \pm 0.17 \mathrm{mag}($ for $\mathrm{E}(\mathrm{B}-\mathrm{V})=0.066 \pm 0.026$ mag as derived from Schlegel et al. 1998 maps).
Both estimates are in good agreement with the value of $(\mathrm{m}-\mathrm{M})_{0}=24.57_{-0.43}^{+0.08} \mathrm{mag}$ found by 


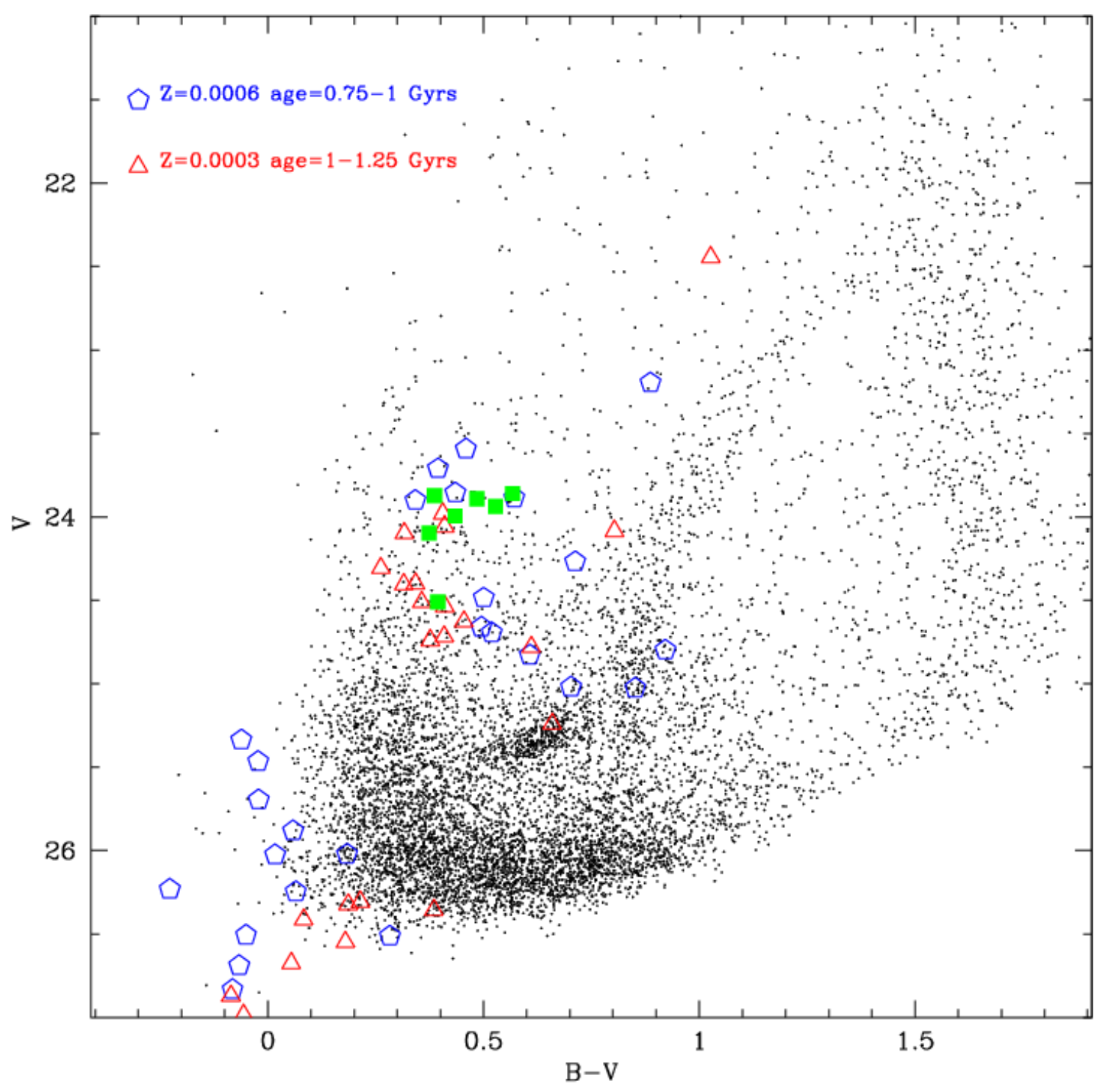

Fig. 14. - Synthetic stellar population allowing to reproduce the number of ACs (green squares) on the observed CMD (see Section 10.2 for details). 
Conn et al. (2012). Comparing the observed CMD with stellar isochrones we find evidence for two different stellar populations in And XIX. One mostly made by old (13 Gyrs) and metal poor $([\mathrm{Fe} / \mathrm{H}]=-$ 1.8 dex) stars which produced the RR Lyrae variables, and the second composed by metal enriched stars $(-1.5<[\mathrm{Fe} / \mathrm{H}]<-1.7$ dex $)$ with ages between 6 and 10 Gyrs. The presence of ACs in And XIX provides hints for a recent episode of star formation in this galaxy. With the use of evolutionary tracks and isochrones, we constrained this formation episode in a epoch between 0.75 and 1.25 Gyrs ago. The ACs are found to follow well a $P W$ relation and are mostly a genuine population belonging to And XIX with no or very little contamination by the M31 halo. The specific frequency of ACs in And XIX is also consistent with the value typical of other dSph galaxies in M31 and the MW. Finally, the spatial distribution of the RGB and HB stars gives indication of the presence of a bar-like structure elongated in the direction of the M31 center.

We warmly thank P. Montegriffo for the development and maintenance of the GRATIS software, G. Beccari for useful suggestions on the estimate of the photometric completeness with ALLFRAME, Flavio Fusi Pecci, Monica Tosi and Carla Cacciari for useful comments and discussions on an earlier version of the paper. Financial support for this research was provided by COFIS ASI-INAF I/016/07/0 and by PRIN INAF 2010 (P.I.: G. Clementini). The LBT is an international collaboration among institutions in the United States, Italy and Germany. LBT Corporation partners are: The University of Arizona o $\mathrm{n}$ behalf of the Arizona university system; Istituto Nazionale di Astrofisica, Italy; LBT Beteiligungsgesellschaft, Germany, representing the Max-Planck Society, the Astrophysical Institute Potsdam, and Heidelberg University; The Ohio State University, and The Research Corporation, on behalf of The University of Notre Dame, University of Minnesota, and University of Virginia. We acknowledge the support from the LBT-Italian Coordination Facility for the execution of observations, data distribution and reduction.

Facilities: LBT.

\section{REFERENCES}

Abazajian, K. N., Adelman-McCarthy, J. K., Ageros, M. A., et al. 2009, ApJS, 182, 543

Bailey, S. I. 1902, Annals of Harvard College Observatory, 38,1

Barning, F. J. M. 1963, Bull. Astron. Inst. Netherlands, 17,22

Bell, E. F., Slater, C. T., \& Martin, N. F. 2011, ApJ, 742, L15

Bertelli, G., Nasi, E., Girardi, L., \& Marigo, P. 2009, A\&A, 508, 355

Bilir, S., Ak, T., Ak, S., Yontan, T., \& Bostanci, Z. F 2013, New Astronomy, 23, 88

Bressan, A., Marigo, P., Girardi, et al. 2012, MNRAS, 427, 127

Brown, T. M., Ferguson, H. C., Smith, E., et al. 2003, ApJ, 592, L17

Brown, T. M., Ferguson, H. C., Smith, E., et al. 2004, AJ, 127, 2738

Cacciari, C., \& Clementini, G. 2003, in Stellar Candles for the Extragalactic Distance Scale, ed. D. Alloin \& W. Gieren (Berlin: Springer), 105

Cacciari, C., Corwin, T. M., \& Carney, B. W. 2005, AJ129, 267

Caputo, F., Castellani, V., \& degl'Innocenti, S. 1995, A\&A, 304, 365

Carretta, E., Bragaglia, A., Gratton, R., D'Orazi, V., \& Lucatello, S. 2009, A\&A, 508, 695

Catelan, M. 2009, Ap\& SS, 320, 261

Cignoni, M., \& Tosi, M. 2010, AdAst2010E, 3

Clement, C. M., \& Rowe, J. 2000, AJ, 120, 2579

Clement, C. M, Muzzin, A., Dufton, Q., et al. 2001, AJ, 122, 2587

Clementini, G., Di Tomaso, S., Di Fabrizio, L., et al. 2000, AJ, 120, 2054

Clementini, G., Gratton, R., Bragaglia, et al. 2003, AJ, 125,1309 
Clementini, G., Contreras Ramos, R., Federici, L., et al. 2009, ApJ, 704, L103

Clementini, G. 2010, in Variable Stars, the Galactic halo and Galaxy Formation, ed. C. Sterken, N. Samus, \& L. Szabados (Moscow: Sternberg Astronomical Institute of Moscow Univ.), 107

Clementini, G., Contreras Ramos, R., Federici, L., et al. 2011, ApJ, 743, 19

Clementini, G., Cignoni, M., Contreras, R., et al. 2012, ApJ, 756, 108

Contreras Ramos, R., Clementini, G., Federici, L., et al. 2013, ApJ, 765, 71

Coe, D., Benitez, N., Sanchez, S.F., et al. 2006, AJ, 132,926

Collins, M. L. M., Chapman, S. C., Rich, R. M., et al. 2013, ApJ768, 172

Conn, A. R., Ibata, R. A., Lewis, G. F., et al. 2012, ApJ, 758, 11

Durrell, P. R., Harris, W. E., \& Pritchet, C. J. 2001, AJ, 121, 2557

Federici. L., Cacciari, C., Bellazzini, M., et al. 2012, A\&A, 544, 155

Gilbert, K., M., Guhathakurta, P., Beaton, R. L., et al. 2012, ApJ, 760, 76

Gratton, R. G., Bragaglia, A., Clementini, G., et al. 2004, A\&A, 421, 937

Harris, W.E. 1996, AJ, 112, 1487

Ibata, R., Martin, N. F., Irwin, M., et al. 2007, ApJ, 671, 1591

Irwin, M. J., Ferguson, A. M. N., Huxor, A. P., et al. 2008, ApJ, 676, L17

Jeffery, E. J., Smith, E., Brown, T. M., et al. 2011, AJ, 141, 171

Kovács, G. 2003, MNRAS, 342, L58

Lee, Y.-W., Demarque, P., \& Zinn, R. 1994, ApJ, 423,248

Lomb, N. R. 1976, Ap\&SS, 39, 447

Mackey, A. D., Huxor, A. P., Martin, N. F., et al. 2013, ApJ, 770, L17
Madore B. F. 1982, ApJ, 253, 575

Mancone, C., \& Sarajedini A. 2008, AJ, 136, 1913

Marconi, M., Fiorentino, G., \& Caputo, F. 2004, A\&A, 417, 1101

Marigo, P., Girardi, L., Bressan, et al. 2008, A\&A, 482,883

Martin, N. F., Ibata, R. A., Irwin, M. J., et al. 2006, MNRAS, 371, 1983

Martin, N. F., McConnachie, A. W., Irwin, et al. 2009, ApJ, 705, 758

Martin, N. F., Slater, C. T., Schlafly, E. F., Morganson, E., \& Rix, H.-W. 2013, ApJ722, 15

Mateo, M., Fischer, P., \& Krzeminski, W. 1995, AJ, 110, 2166

McConnachie, A. W., Huxor, A., Martin, N. F., et al. 2008, ApJ, 688, 1009

McConnachie, A. W., Irwin, M. J., Ibata, R. A., et al. 2009, Nature, 461, 66

McConnachie, A. W. 2012, AJ144, 4

Oosterhoff, P. T. 1939, The Observatory, 62, 104

Piersimoni, A. M., Bono, G., \& Ripepi, V. 2002, AJ, 124, 1528

Pietrinferni, A., Cassisi, S., Salaris, \& M. Castelli, F. 2004, ApJ, 612, 168

Piotto, G., King, I. R., Djorgovski, S. G., \& et al. 2002, A\&A, 391, 945

Pritzl, B. J., Armandroff, T. E., Jacoby, G. H., \& Da Costa, G. S. 2002, AJ, 124, 1464

Pritzl, B. J., Jacoby, G. H., \& Da Costa, G. S. 2004, AJ, 127, 318

Pritzl, B. J., Armandroff, T. E., Jacoby, G. H., \& Da Costa, G. S. 2005, AJ, 129, 2232

Richardson, J. C., Irwin, M. J., McConnachie, A. W., et al. 2011, ApJ, 732, 76

Ripepi, V., Marconi, M., Moretti, M. I., et al. 2013, MNRAS, resubmitted

Sarajedini, A., Mancone, C. L., Lauer, T. R., et al. 2009, AJ, 138, 184 
Scargle, J. D. 1982, ApJ, 263, 835

Schlegel, D. J., Finkbeiner, D. P., \& Davis, M. 1998, ApJ, 500, 525

Searle, L., \& Zinn, R. 1978, ApJ, 225, 357

Slater, C. T., Bell, E. F., \& Martin, N. F. 2011, ApJ, 742, L14

Soszynski, I., Udalski, A., Szymanski, M. K., et al. 2008, AcA, 58, 293

Soszynski, I., Poleski, R., Udalski, A., et al. 2008, AcA, 58, 163

Starkerburg, E., Hill, V., Tolstoy, E., et al. 2010, A\&A, 513, 34

Stetson, P. B. 1987, PASP, 99, 191

Stetson, P. B. 1992, JRASC, 86, 71

Stetson, P. B. 1994, PASP, 106, 250

van den Bergh, S. 1975, StarsandStellarSystems, 9, 509, ed. A. R. Sandage, M. Sandage, and J. Kristian (Chicago: University of Chicago Press)

van den Bergh, S. 1993, MNRAS, 262, 588

Wen, Z. L., \& Han, J. L. 2013, arXiv1301.0871W

Yang, S.-C. \& Sarajedini, A. 2012,MNRAS, 419, 1362

Zucker, D. B., Kniazev, A. Y., Bell, E. F., et al. 2004, ApJ, 612, L121

Zucker, D. B., Kniazev, A. Y., Martinez-Delgado, D., et al. 2007, ApJ, 659, L21

This 2-column preprint was prepared with the AAS LATEX macros v5.2. 